\title{
New 6- and 7-heterocyclyl-1H-indole derivatives as potent tubulin assembly and cancer cell growth inhibitors
}

Giuseppe La Regina a, Ruoli Bai b, Antonio Coluccia a, Valentina Naccarato a, Valeria Famiglini a, Marianna Nalli a, Domiziana Masci a, Annalisa Verrico c, Paola Rovella c, Carmela Mazzoccoli d, Eleonora Da Pozzo e, Chiara Cavallini e, Claudia Martini e, Stefania Vultaggio f, Giulio Dondio g, Mario Varasi f, Ciro Mercurio f, Ernest Hamel b, Patrizia Lavia c, Romano Silvestri a, *

a Department of Drug Chemistry and Technologies, Sapienza University of Rome, Laboratory Affiliated to Istituto Pasteur Italia e Fondazione Cenci Bolognetti, Piazzale Aldo Moro 5, I00185 Roma, Italy

bScreening Technologies Branch, Developmental Therapeutics Program, Division of Cancer Treatment and Diagnosis, Frederick National Laboratory for Cancer Research, National Cancer Institute, National Institutes of Health, Frederick, MD 21702, United States

c Institute of Molecular Biology and Pathology (IBPM), CNR Consiglio Nazionale Delle Ricerche, C/o Sapienza University of Rome, Via Degli Apuli 4, I-00185 Roma, Italy

d Laboratorio di Ricerca Pre-Clinica e Traslazionale, Istituto di Ricovero e Cura a Carattere Scientifico (IRCCS), Centro di Riferimento Oncologico Della Basilicata, Via Padre Pio 1, I-85028, Rionero in Vulture, Italy

e Department of Pharmacy, University of Pisa, Via Bonanno Pisano 6, I-56126 Pisa, Italy

f Experimental Therapeutics IFOM-the FIRC Institute of Molecular Oncology Foundation, Via Adamello 16, 20139 Milan, Italy g APHAD, Via Della Resistenza 65, 20090 Buccinasco MI, Italy

\begin{abstract}
We designed new 3-arylthio- and 3-aroyl-1H-indole derivatives 3e22 bearing a heterocyclic ring at position 5, 6 or 7 of the indole nucleus. The 6- and 7-heterocyclyl-1H-indoles showed potent inhibition of tubulin polymerization, binding of colchicine to tubulin and growth of MCF-7 cancer cells. Compounds 13 and 19 inhibited a panel of cancer cells and the $\mathrm{NCl} / A D R-R E S$ multidrug resistant cell line at low nanomolar concentrations. Compound 13 at $50 \mathrm{nM}$ induced $77 \% \mathrm{G} 2 / \mathrm{M}$ in HeLa cells, and at $20 \mathrm{nM}$ caused 50\% stable arrest of mitosis. As an inhibitor of HepG2 cells (IC50 1/4 20 nM), 13 was $4-$ fold superior to 19. Compound 13 was a potent inhibitor of the human U87MG glioblastoma cells at nanomolar concentrations, being nearly one order of magnitude superior to previously reported arylthioindoles. The present results highlight 13 as a robust scaffold for the design of new anticancer agents.
\end{abstract}

\section{Introduction}

Microtubules (MTs) are hollow, cylindrical, filamentous structures made of dimerized a- and b-tubulin subunits and are characterized by a highly dynamic equilibrium involving polymerization, where tubulin dimers bind non-covalently to aMT, and depolymerization to tubulin dimers [1]. MTs regulate key cellular functions, such as cell growth and division, intracellular trafficking, preservation of the architecture of the cell, and motility. Due to these key roles, disrupting the dynamic equilibrium of MTs at either the tubulin assembly or MT disassembly level, results in a fatal cellular event. Cancer cells are characterized by a high rate of cell division: hence, the strategy of inducing cell death through an interference with the dynamics of MTs has proved successful for the design of effective antitumor drugs [2,6].

MT binding agents fall into two main groups: (i) inhibitors of tubulin polymerization, including colchicine [7,8], combretastatin A-4 (CSA4) [9] (Chart 1), vincristine (VCR), vinorelbine (VRB) and vinblastine (VBL); and (ii) MT stabilizers, including taxoids and epothilones. The stabilizers stimulate MT polymerization and stabilization at high concentrations, whereas at lower concentrations paclitaxel (PTX) inhibits MT dynamics with little effect on the proportion of tubulin in polymer [10]. Taxoids and epothilones bind at a luminal site on the b-subunit [11,12] following entry into the MT through pores in its wall [13] that are shaped by various tubulin subunits on the MT surface. Some evidence indicates a transient binding of MT stabilizers at a specific pore site.

Despite considerable clinical successes [14,15], the anticancer therapies based on tubulin binding agents still have limitations perhaps due to multi-drug resistance (MDR), toxicity and unwanted side effects [16,17]. Therefore, there is a quest for new effective MT inhibitors with fewer side effects to become components of improved anticancer treatments $[18,19]$.

Currently, there are no US Food and Drug Administration approved anticancer drugs on the market binding at the colchicine site [20]. Compared to other tubulin agents, these compounds (i) have shown less transporter mediated drug resistance in preclinical studies [21], (ii) are minimally affected by the overexpression of blll-tubulin [22], and (iii) have generally better water solubility [9]. Improvements of activity of colchicine site binding agents by replacement or introduction of heterocyclic ring(s) to the parent scaffold have been reported [16,23,24]. Accordingly, as tubulin polymerization inhibitors, 2-heterocyclyl-3-arylthio-1H-indole (2-HATI) derivatives were more effective than the corresponding 2-aryl-3-arylthio-1H-indole counterparts in both the sulfur and aroyl series [25]. For example, 2(thiophen-2-yl)-3-[(30,40,50-trimethoxyphenyl)thio]-1H-indole (1) inhibited tubulin assembly with an IC50 of $0.74 \mathrm{mM}$ and MCF-7 cancer cell growth with an IC50 of $39 \mathrm{nM}$ [26]. Compound 1 was superior to VRB, VBL, and PTX as an inhibitor of the P-glycoprotein (Pgp) overexpressing $\mathrm{NCl}$ / adriamycin-resistant (ADR-RES) MDR cell line and showed satisfactory metabolic stability. Recently, introduction of halogen or methoxy substituent(s) at positions $4 \mathrm{e} 7$ of 3-arylthio- $1 \mathrm{H}$-indoles (ATIs) bearing the phenyl at position 2 of the indole provided potent tubulin polymerization inhibitors (for example 2) [27]. 
Herein we report the synthesis of new indole derivatives 3e22 bearing a five-membered heterocyclic ring at position 5, 6 or 7 of the indole ring (Chart 1 and Table 1). Our findings demonstrate that the new derivatives are potent anticancer agents, superior to the corresponding reference derivative 1 [26]. Compounds 13 and 19 inhibited the HT29, HCT116, HepG2, T98G, U87MG, U343G and NCl/ ADR-RES cell lines with IC50 values in the nanomolar range. Compound 13 was superior to the previously reported compounds [26] as an inhibitor of HepG2 and glioblastoma cells.

\section{Chemistry}

\subsection{Synthetic procedures}

Arylthioindoles $3 e 7,9,11,13,15,19$ and 37 were prepared according to our previously reported venting-while-heating microwave (MW)-assisted procedure [28] by treating the appropriate indole with bis(3,4,5-trimethoxyphenyl)disulfide [29] in the presence of sodium hydride in anhydrous N,N-dimethylformamide (DMF) at 130 _ for 2 min (120 W) (Scheme 1). Reaction of the indole with 3,4,5-trimethoxybenzoyl chloride in the presence of diethylaluminum chloride in dichloromethane at $10 \mathrm{C}$ for $2 \mathrm{~h}$ furnished methanones 8, 10, 12 and 14. Derivatives 16e18, 20e22, 30e32, 35 and 36 were synthesized by a coupling reaction of the appropriate indole with the boronic derivative in the presence of tris(dibenzylideneacetone)dipalladium(0) (Pd2dba3), 2-dicyclohexylphosphino-20,60-dimethoxybiphenyl (SPhos) and potassium phosphate tribasic in 1-butanol at 100 _C for $15 \mathrm{~h}$. A palladium(II) acetate ( $\mathrm{Pd}(\mathrm{OAc}) 2)$-catalyzed reaction of 5-iodo-1Hindole (24) with 2-furanboronic or 2-thienylboronic acid in the presence of tri(o-tolyl)phosphine ( $\mathrm{P}(\mathrm{o}$-tol)3) and potassium phosphate tribasic in ethanol/toluene at $80 \mathrm{C}$ for $2 \mathrm{~h}$ gave the corresponding heteroaryl indoles 27 and 29 , respectively. $\mathrm{MW}$-assisted treatment of indole 24 with 3-furanboronic acid pinacol ester in the presence of $\mathrm{Pd}(\mathrm{OAc}) 2$ and potassium carbonate in methylpyrrolidone/ water at $110 \mathrm{C}$ for $15 \mathrm{~min}(200 \mathrm{~W})$ furnished 5-(furan- 2-yl)-1H-indole (28). Compound 38 was prepared by MW-assisted Friedel-Crafts reaction of 7-iodo-1H-indole (26) with 3,4,5trimethoxybenzoyl chloride in the presence of anhydrous aluminum chloride in 1,2-dichloroethane at 110 _ for 2 min (150 W).

\section{Results and discussion}

\subsection{Inhibition of tubulin polymerization}

We synthesized arylthioindole and aroylindole derivatives 3-22 to explore the effects of five-membered heterocyclic rings at position 5, 6 or 7 of the indole nucleus (Table 1). Ten new derivatives $(3,7,8,10$ e15, and 19) inhibited tubulin polymerization with IC50 values at submicromolar concentrations, six compounds (4e6, 16, 17 and 21) were in the 1.0e2.0 mM range, as compared with colchicine (IC50 $1 / 43.2 \mathrm{mM}$ ) and CSA4 (IC50 $1 / 41.0 \mathrm{mM}$ ). With the exception of 9 , the most potent tubulin polymerization inhibitors (7, 8 and 10e14) were characterized by the presence of the heterocyclic ring at position 6 of the indole; among others, compound 3, with the heterocyclic ring at position 5 of the indole, and 16, 17 and 21, with the heterocyclic ring at position 7, showed IC50 values < $1.0 \mathrm{mM}$. As tubulin assembly inhibitors, the compounds bearing the furan-2-yl or the thiophen-2-yl ring were generally superior to the corresponding furan-3yl-/thiophen-3-yl derivatives (compare 3 with 4, 5 with 6, 7 with 9, 15 with 17, and 19 with 21).

Replacement of the sulfur bridging atom with the carbonyl group also provided potent tubulin assembly inhibitors. The presence of the heterocycle at position 7 of the indole conferred significant differences of inhibition of tubulin polymerization among arylylthio/aroyl derivatives (compare 15 with 16, 17 with 18, 19 with 20, and 21 with 22), while compounds 7e14 with the heterocycle at position 6 were almost equipotent, except 9 and 10, with 12 (IC50 1/4 $0.38 \mathrm{mM}$ ) being the most potent tubulin polymerization inhibitor among the new compounds.

\subsection{MCF-7 breast cancer cell growth}

Several new indole derivatives inhibited the growth of human MCF-7 nonmetastatic breast cancer epithelial cells with IC50 values at nanomolar concentrations (Table 1). Structure-activity relationship (SAR) analysis show three distinct groups: (i) introduction of the heterocyclic ring at position 5 of the indole had a relatively weak effect on inhibition of MCF-7 cell growth, with IC50 values ranging from 70 (3) to $200 \mathrm{nM}(4,6)$; (ii) compounds 7e14, bearing the heterocyclic ring at position 6 of the indole, all were highly potent MCF-7 cell growth inhibitors, with IC50 values ranging from 4.3 (10) to 18 (14) nM, with five compounds (8 and 10e13) having single digit nanomolar IC50 values; (iii) the indoles with the heterocycle at position 7 showebridging group: the 7-heterocyclyl-3-arylthio-1H-indoles 15, 17, 19 and 21 (IC50s ranging from 15 to $38 \mathrm{nM}$ ) were more than one order of magnitude superior to the corresponding aroyl derivatives 16 , 18,20 and 22.

A SAR summary of tubulin polymerization inhibition and inhibition of MCF-7 cell growth of compounds $3 e 22$ is depicted in Chart 2. It should be noted that the SARs differ from those of the 2- phenyl-1H-indoles [27]. Compounds $3 e 6$ with the heterocyclic ring at position 5 showed moderate inhibition of MCF-7 cell growth; compounds 7e14, bearing the heterocycle at position 6 of the indole, all were potent MCF-7 cell growth inhibitors and showed comparable IC50 values; derivatives 15, 17, 19 and 22, with the heterocyclic ring at position 7 and the sulfur atom bridging the two aromatic ring systems, were substantially superior to the corresponding aroyl compounds (16, 18, 20 and 22).

\subsection{Inhibition of the binding of $[3 \mathrm{H}]$ colchicine to tubulin}

Compounds $3 \mathrm{e} 22$ were also examined for potential inhibition of the binding of $[3 \mathrm{H}]$ colchicine to tubulin (Table 1 ). Thirteen compounds, 3, 5, 7e17, and 19 yielded $>75 \%$ inhibition of the binding reaction. Interestingly, all the indoles with the heterocycle at position 7 were strong inhibitors of $[3 \mathrm{H}]$ colchicine binding, with $13(97 \%$ inhibition) nearly as 
potent as CSA4 (98\% inhibition). In general, good correlation between [3H]colchicine binding and MCF- 7 cell growth inhibitionwas observed: compounds which inhibited $[3 \mathrm{H}]$ colchicine binding $>90 \%$, inhibited the growth of the MCF-7 cancer cell with IC50s_ $15 \mathrm{nM}$; compounds with 80e90\% inhibition of [3H]colchicine binding inhibited the growth of the MCF-7 cancer cell line with IC50s in the range of $24 \mathrm{e} 70 \mathrm{nM}$; except for 21 , compounds with $70 \mathrm{e} 80 \%$ inhibition of $[3 \mathrm{H}]$ colchicine binding inhibited the growth of the MCF-7 cancer cells with IC50s in the range of $150 \mathrm{e} 350 \mathrm{nM}$.

\subsection{Molecular modeling studies}

Compounds 6, 13 and 19, representative members of the heterocyclic substitutions at position 5-, 6- or 7- of the indole nucleus, were docked into the tubulin-DAMA-colchicine complex (PDB code 1SA0) [8] by following our previously reported procedure [30]. The docking studies were also performed for five newly available tubulin crystal structures [31]. The docking results in the different tubulin structures revealed a consistent binding mode for compounds bearing the heterocyclic ring at position 5-, 6- or 7- of the indole: (i) the 3,4,5-trimethoxyphenyl moiety formed an $\mathrm{H}$-bond with the Cys241b side chain and hydrophobic contacts with Leu248b and Leu255b; (ii) the indole established hydrophobic contacts with Asn258b and Met259b; (iii) the heterocyclic ring of 6, 13 and 19 at position 5, 6 or 7 of the indole, respectively, arranged hydrophobic interactions with Met259b, Lys353b, Ala180a and Val181 in the same cleft of the colchicine site (Figs. 1 and 2).

\subsection{Inhibition of growth of glioblastoma T98G, U87MG and U343MG cell lines}

Treatments of T98G (human glioblastoma), U87MG (human glioblastoma-astrocytoma) and U343MG (human glioblastomaastrocytoma) cells with increasing concentrations of 13 and 19 significantly inhibited cell growth in a dosedependent manner (Table 2 and Fig. 1SD-3SD, supplementary data). The IC50 values were calculated taking into account the relative cellular doubling times [32,33] of $48 \mathrm{~h}$ for the T98G and U87MG cells and of $72 \mathrm{~h}$ for the U343MG cells. Compound 13 potently inhibited the glioblastoma cells at nanomolar concentrations. Arylthioindoles with the imidazole-1-yl or pyridin-4-yl heterocyclic ring at position 2 of the indole were weaker inhibitors of glioblastoma U87MG cells than were 13 and 19, even though such compounds exhibited potent inhibition of the MCF-7 cancer cell growth [26].

\subsection{Inhibition of HT29, HCT116 and HepG2 cancer cell growth}

Compounds 13 and 19 were evaluated as growth inhibitors of the human cell lines HT29 (colon adenocarcinoma), HCT116 (colon carcinoma), and HepG2 (hepatocellular carcinoma) using PTX as reference compound (Table 2). Compounds 13 and 19 showed strong inhibition of the HT29 and HCT116 cell lines. As inhibitors of the HepG2 cells, 13 and 19 were more effective than PTX (IC50 1/4 $2660 \mathrm{nM})$.

\subsection{Inhibition of MV4-11, THP-1, A-549 and PC3 cell lines}

Compounds 13 and 19 were evaluated as inhibitors of MV4-11 (leukemia, acute myeloid), THP1 (leukemia, acute monocytic), A549 (lung carcinoma) and PC3 (prostate carcinoma) cancer cells. Compound 13 inhibited MV4-11, THP-1, A-549 and PC3 cells at single digit nanomolar concentration, while 19 was consistently less active (Table 2).

\subsection{MDR cell lines}

Compounds 13 and 19, representative of the 6- and 7- heterocyclyl series, respectively, were compared with CSA4, VRB, VLB and PTX in the ovarian carcinoma cell lines OVCAR-8 and its cognate Pgp overexpressing line NCl/ADR-RES (Table 3). In contrast to CSA4, the standard agents VRB, VLB and PTX showed weak inhibition of the MDR line NCI/ADR-RES. The IC50 values of 13 and 19 for the MCF-7 cells, repeated in this study, resembled those obtained in the studies summarized in Table 1.

\subsection{Compounds 13 and 19 induce cell cycle arrest in the G2/M phase in HeLa cells}

We compared cell cycle progression in HeLa cells exposed to 20, 50 and 100 nM 13 and 19, using 20 nM VBL as a reference compound (a concentration that effectively arrests cells in mitosis [27]) and DMSO as a control. Cells were treated for $24 \mathrm{~h}$ to cover the average duration of an entire cell cycle, then harvested, incubated with propidium iodide (PI) and analyzed for their genomic content by FACS analysis. Representative cell cycle profiles are shown in Fig. 3A, left panel; data from three experiments are quantified in the graph in Fig. 3A, right panel. Both molecules inhibited cell cycle progression in a dose-dependent manner but with different effectiveness: treatment with $100 \mathrm{nM} 19$ yielded a substantial fraction (over 71\%) of the cell population in the G2/M phase, whereas lower doses had no significant effect; compound 13 was already partly effective at $20 \mathrm{nM}$ and induced substantial G2/M arrest at $50 \mathrm{nM} \mathrm{(77 \%} \mathrm{arrest).}$

Immunofluorescence (IF) analysis of 13 and 19-treated HeLa cell cultures confirmed that treatment with $20 \mathrm{nM} 13$ (24 h) induced arrest in mitosis in over $50 \%$ of all cells, similar to the effect of VBL. The mitotic arrest was stable, and only $15 \%$ of the cell population included multinucleated cells, resulting from mitotic "slippage" and interphase re-entry with unsegregated, or randomly segregating, chromosomes. Compound 19 induced mitotic arrest with lower effectiveness than 13: (i) $100 \mathrm{nM} 19$ arrested 50\% of the cell population in mitosis, compared with $20 \mathrm{nM} 13$ or VBL; (ii) the induction of mitotic arrest by 19 was not fully sustained. The higher accumulation of cells in mitosis observed with $100 \mathrm{nM} 19$ was accompanied by induction of a relevant fraction (around 32\%) of multinucleated cells: this suggests that cells that reached mitosis arrested only transiently, and eventually resumed mitotic progression with an inefficient mitotic apparatus, generating multinucleated cellular offspring (Fig. 3B). We wondered whether cell cycle arrest by 13 and 19 was paralleled by cell death induction, as is the case with classical MTtargeting drugs. Cultures treated for $24 \mathrm{~h}$ were 
incubated with annexin $\mathrm{V}$ to identify cells with damaged plasma membranes committed to death. Both compounds activated cell death parallel to arresting cell cycle progression; again, 19 was effective when used at $100 \mathrm{nM}$, whereas $20 \mathrm{nM} 13$ was already partly effective and reached the same death-inducing capacity as VBL at the $50 \mathrm{nM}$ dose (Fig. $3 \mathrm{C}$ ). The tubulin inhibitory effects of 13 and 19 at the cytological level were evaluated by IF analysis (Fig. 4). These assays provided a visual demonstration of the differential effectiveness of the two compunds.

Compund 19 at 20 or $50 \mathrm{nM}$ induced a mixture of apparently normal, or fragmented or multipolar spindles in variable proportions (examples are shown in Fig. 4A, phenotypes are quantified in Fig. 4C); $100 \mathrm{nM} 19$ largely induced small formations of radially arranged short microtubules that failed to elongate (labeled as "MT asters", Fig. 4A, central row). Compound 13 had a more pronounced effect at all tested concentrations, and no cell showed a normal or only weakly affected spindle. Even with 13 at $20 \mathrm{nM}$, most mitotic cells displayed multipolar (Fig. 4B, top row) or fragmented spindles (Fig. 4B, central row); at higher concentration, $50 \mathrm{nM}$ or above, 13 inhibited MT formation altogether, with sparse tubulin foci in virtually all treated cells (Fig. 4B, bottom row). Representative phenotypes are shown in Fig. $4 \mathrm{~A}$ and B and quantified in Fig. 4C.

In summary, both 13 and 19 arrested cells in mitosis, with formation of a defective mitotic apparatus, yet the two compounds showed differential effectiveness. Compound 13 induced the inhibition of MT polymerization to a greater extent than 19 , and it showed a full inhibitory effect on tubulin polymerization in cells at $50 \mathrm{nM}$, comparable to the effect of VBL. Compound 19 induced milder inhibition: it essentially affected the mitotic spindle structural organization but did not fully inhibit tubulin polymerization in cells below $100 \mathrm{nM}$. The parallel observation that 13 was a more effective inducer of cell death than 19 correlated with cellular data on microtubule disruption and with inhibition of $[3 \mathrm{H}]$ colchicine binding.

\subsection{Effects of 13 or 19 on viability of HepG 2 cells}

HepG2 cells were treated with different concentrations of 13 or 19 and analyzed by the MTT assay. Treatment with compound 19 for $48 \mathrm{~h}$ caused a dose-dependent decrease of cell viability: at $80 \mathrm{nM}$ nearly $30 \%$ of cells displayed reduced viability as compared to untreated cells (Fig. 4SD, panel A, supplementary data). The dose-dependent decrease of cell viability on treatment with 13 became significant at $20 \mathrm{nM}$, nearly $40 \%$ of cells were affected (Fig. 4SD, panel B, supplementary data). These experiments indicated $80 \mathrm{nM} 19$ and $20 \mathrm{nM} 13$ are optimal concentrations to induce significant modifications in cell viability but, at the same time, preserving a sufficient proportion of the cell population to permit further analysis. At such concentrations, 13 and 19 caused a marked impairment of HepG2 cell growth after a $48 \mathrm{~h}$ treatment. The cellcycle blocker p21Cip1/Waf1 was significantly up-regulated in HepG2 treated cells at these doses. After $80 \mathrm{nM} 19$ (Fig. 5SD, panel A, supplementary data) or $20 \mathrm{nM} 13$ (Fig. 5SD, panel B, supplementary data) treatments, the transcript levels of p21Cip1/Waf1, assessed by qRT-PCR, were increased by 1.5- and 3.9-fold, respectively, as compared with untreated cells.

\subsection{Evaluation of the drug-like properties of 13 and 19}

Drug like properties of compounds 13 and 19 were predicted through the most common descriptors of drug-likeness (Table 4). Oral absorption according to Lipinski's rule of five [34] and Veber's rule [35] was estimated by FAF drug server [36]. We referred to the $3 / 75$ rule ( $\log P>3$ and topological PSA < $75 \AA 2$ ) [37] to estimate compound toxicity. Derivatives 13 and 19 properly fitted with both Lipinsky and Veber's rules, suggesting a potential good absorption after oral administration. Furthermore, a low likelihood of in vivo toxicological outcome was inferred by the 3/75 rule (Fig. 5).

\section{Conclusions}

We designed new 3-arylthio- and 3-aroyl-1H-indole derivatives 3-22 bearing a heterocyclic ring at position 5, 6 or 7 of the indole nucleus. Ten new derivatives inhibited polymerization of purified tubulin with IC50 values at submicromolar concentrations. Seven of the new indole derivatives inhibited the growth of MCF-7 cells with IC50 values 10 nM. Inhibition of cell growth showed good correlation with inhibition of $[3 \mathrm{H}]$ colchicine binding to tubulin. Two representative highly potent members of the 6- and 7-heterocyclyl-1H-indoles, 13 and 19, inhibited an extensive panel of cancer cell lines, including the Pgp overexpressing NCI/ADR-RES cell line, with nanomolar IC50s. In cell cycle analysis, compound 13 became effective at $20 \mathrm{nM}$ and induced $77 \%$ G2/M arrest at $50 \mathrm{nM}$, whereas 19 accumulated $71 \%$ cells in the G2/M phase at $100 \mathrm{nM}$. Compound 13 extensively inhibited cellular tubulin polymerization at $50 \mathrm{nM}$, and its activity was comparable to that of VBL. Compound 19 at $100 \mathrm{nM}$ affected mitotic spindle structural organization but did not fully inhibit cellular tubulin polymerization. As inhibitors of HepG2 cells, 13 and 19 (IC50 values of $20 \mathrm{nM}$ and $80 \mathrm{nM}$, respectively) were distinctly superior to PTX. Compound 13 was also a potent inhibitor of the U87MG glioblastoma cell line at nanomolar concentrations [41] being almost one order of magnitude more active than the previously reported arylthioindoles with the imidazole-1-yl or pyridin-4-yl ring at position 2 of the indole nucleus [26]. Analysis of the gene expression levels of p21Cip1/Waf1, the cell cycle blocker, indicated that at sub-cytotoxic concentrations 13 caused a decrease in cell growth with strong up-regulation of p21Cip1/Waf1 [42].

In conclusion, these new indoles are potent inhibitors of tubulin polymerization and cancer cell growth, including human liver carcinoma and glioblastoma cells. The introduction of the heterocyclic ring at position 5, 6 or 7 of the 3-(30,40,50trimethoxyarylthio)-1H-indole and 3-(30,40,50-trimethoxyaroyl)-1H-indole scaffold was a productive strategy for the 
design of new effective anticancer agents, with compound 13 as a robust lead agent. Based on SAR findings, the synthesis of new analogues is in progress in our laboratory, and the results will be reported in due course.

\section{Experimental protocols}

\subsection{Chemistry}

All reagents and solvents were handled according to the material safety data sheet of the supplier and were used as purchased without further purification. 5-Chloro-1H-indole (23), 5-iodo-1Hindole (24), 6-bromo-1H-indole (25), 7-iodo$1 \mathrm{H}$-indole (26), 6- (thiophen-2-yl)-1H-indole (33) and 6-(thiophen-3-yl)-1H-indole (34) were commercially available. $\mathrm{MW}$-assisted reactions were performed on a CEM Discover SP single-mode reactor equipped with an Explorer 72 autosampler, controlling the instrument settings by PC-running CEM Synergy 1.60 software. Closed vessel experiments were carried out in capped $\mathrm{MW}$-dedicated vials $(10 \mathrm{~mL})$ with a cylindrical stirring bar (length $8 \mathrm{~mm}$, diameter $3 \mathrm{~mm}$ ). Stirring, temperature, irradiation power, maximum pressure (Pmax), pressure set point, times at set point, delta pressure, PowerMAX (simultaneous cooling-while-heating), ActiVent (simultaneous venting-while-heating), and ramp and hold times were set as indicated. Reaction temperaturewas monitored by an external CEM fiber optic temperature sensor. After completion of the reaction, the mixture was cooled to 25 _ C via air-jet cooling. Organic solutions were dried over anhydrous sodium sulfate. Evaporation of solvents was carried out on a Büchi Rotavapor R-210 equipped with a Büchi V-850 vacuum controller and a Büchi V-700 vacuum pump. Column chromatography was performed on columns packed with silica gel from Macherey-Nagel (70e230 mesh). Silica gel thin layer chromatography (TLC) cards from Macherey-Nagel (silica gel precoated aluminum cards with fluorescent indicator visualizable at $254 \mathrm{~nm}$ ) were used for TLC. Developed plates were visualized with a Spectroline ENF 260C/FE UV apparatus. Melting points (mp) were determined on a Stuart Scientific SMP1 apparatus and are uncorrected.

Infrared (IR) spectra were recorded on a PerkinElmer Spectrum 100 FT-IR spectrophotometer equipped with a universal attenuated total reflectance accessory and IR data acquired and processed by PerkinElmer Spectrum 10.03.00.0069 software. Band position and absorption ranges are given in cm_1. Proton nuclear magnetic resonance (1H NMR) spectra were recorded with a Varian Mercury $(300 \mathrm{MHz})$ or a Bruker Avance $(400 \mathrm{MHz})$ spectrometer in the indicated solvent, and the corresponding fid files were processed by MestreLab Research SL MestreReNova 6.2.1e769 software.

Chemical shifts are expressed in $d$ units (ppm) from tetramethylsilane. Mass spectra were recorded on a Bruker Daltonics MicroTOF LC/MS mass spectrometer equipped with a positive ion ESI source. Compound purity was checked by high pressure liquid chromatography (HPLC). Purity of tested compounds was found to be $>95 \%$. The HPLC system used (Thermo Fisher Scientific Inc. Dionex UltiMate 3000) consisted of an SR-3000 solvent rack, a LPG-3400SD quaternary analytical pump, a TCC-3000SD column compartment, a DAD-3000 diode array detector, and an analytical manual injection valve with a $20 \mathrm{~mL}$ loop. Samples were dissolved in acetonitrile $(1 \mathrm{mg} / \mathrm{mL})$. HPLC analysis was performed by using a Thermo Fisher Scientific Inc. Acclaim $120 \mathrm{C} 18$ column ( $\left.5 \mathrm{~mm}, 4.6 \mathrm{~mm} \_250 \mathrm{~mm}\right)$ at $25 \pm 1$ _ with an appropriate solvent gradient (acetonitrile/water), flow rate of $1.0 \mathrm{~mL} / \mathrm{min}$ and signal detector at 206, 230, 254 and $365 \mathrm{~nm}$. Chromatographic data were acquired and processed by Thermo Fisher Scientific Inc. Chromeleon 6.80 SR15 Build 4656 software.

\subsubsection{General procedure for the synthesis of derivatives $3 e 7,9,11,13,15,19$ and 37.}

Example. 5-(Furan-2-yl)-3-((3,4,5- trimethoxyphenyl)thio)-1H-indole (3)

A mixture of indole 27 (183 mg, $1 \mathrm{mmol})$, bis(3,4,5- trimethoxyphenyl)disulfide [29] (438 $\mathrm{mg}, 1.1 \mathrm{mmol})$, and sodium hydride ( $88 \mathrm{mg}, 2.2 \mathrm{mmol}$; $60 \%$ in mineral oil) in anhydrous DMF ( $3 \mathrm{~mL}$ ) was placed into the MW cavity (closed vessel mode, Pmax $1 / 4250$ psi). Starting MW irradiation of 120W was used, the temperature being ramped from 25 to 130 C, while rapidly stirring and venting (pressure set point: 100 psi; times at set point: 100; delta pressure: 20 psi). Once 130 _ $\mathrm{C}$ was reached, taking about $1 \mathrm{~min}$, the reaction mixture was held at this temperature for 2 min. The mixture was diluted with water and extracted with ethyl acetate. The organic layer was washed with brine and dried. Removal of the solvent gave a residue that was purified by column chromatography (silica gel, $n$-hexane:ethyl acetate $1 / 4$ 3:2 as eluent) to give 3 (122 mg, yield 32\%), mp 118e120_C (from ethanol). 1H NMR (DMSO-d6, $400 \mathrm{MHz}$ ): d 3.98 (s, 9H), 6.82 (s, 2H), $6.95(\mathrm{~s}, 1 \mathrm{H}), 7.22(\mathrm{~d}, \mathrm{~J} \mathrm{1} / 41.7 \mathrm{~Hz}, 1 \mathrm{H}), 7.93 \mathrm{e} 7.96(\mathrm{~m}, 2 \mathrm{H}), 8.07(\mathrm{~s}, 1 \mathrm{H}), 8.17(\mathrm{~s}, 1 \mathrm{H}), 8.22(\mathrm{~s}, 1 \mathrm{H}), 12.20 \mathrm{ppm}$ (br s, disappeared after treatment with D2O, 1H). IR: n $3448 \mathrm{~cm} \_1$. MS (ESI): 382.4 (MHp). C21H19NO4S (381.45).

\subsubsection{5-(Furan-3-yl)-3-((3,4,5-trimethoxyphenyl)thio)-1H-indole (4)}

Synthesized as 3 starting from 28. Yield 20\%, mp 125e130_C (from ethanol). 1H NMR (DMSO-d6, 400 MHz): d 3.57 (s, $3 \mathrm{H}), 3.59(\mathrm{~s}, 6 \mathrm{H}), 6.44(\mathrm{~s}, 2 \mathrm{H}), 6.89 \mathrm{e} 6.91(\mathrm{~m}, 1 \mathrm{H}), 7.45 \mathrm{e} 7.47(\mathrm{~m}, 2 \mathrm{H}), 7.61 \mathrm{e} 7.65(\mathrm{~m}, 1 \mathrm{H}), 7.70(\mathrm{~d}, \mathrm{~J} / \mathrm{4} 1.7 \mathrm{~Hz}, 1 \mathrm{H}), 7.77(\mathrm{~s}$, $1 \mathrm{H}), 8.05 \mathrm{e} 8.10(\mathrm{~m}, 1 \mathrm{H}), 11.70 \mathrm{ppm}$ (br s, disappeared after treatment with D2O, 1H). IR: $\mathrm{n} 3425 \mathrm{~cm} \_1 . \mathrm{MS}$ (ESI): 382.2 (MHp). C21H19NO4S (381.45).

\subsubsection{5-(Thiophen-2-yl)-3-((3,4,5-trimethoxyphenyl)thio)-1Hindole (5)}

Synthesized as 3 starting from 29. Yield 31\%, mp 115e120_C (from ethanol). 1H NMR (CDCl3, $400 \mathrm{MHz}): \mathrm{d} 3.69$ (s, 6H), $3.78(\mathrm{~s}, 3 \mathrm{H}), 6.44(\mathrm{~s}, 2 \mathrm{H}), 7.00 \mathrm{e} 7.07(\mathrm{~m}, 1 \mathrm{H}), 7.24(\mathrm{dd}, \mathrm{J} 1 / 42.9$ and $7.1 \mathrm{~Hz}, 1 \mathrm{H}), 7.28(\mathrm{~s}, 1 \mathrm{H}), 7.44(\mathrm{~d}, \mathrm{~J} \mathrm{1} / 48.5 \mathrm{~Hz}, 1 \mathrm{H}), 7.52$ (d, J 1 $1 / 42.6 \mathrm{~Hz}, 1 \mathrm{H}), 7.55 \mathrm{e} 7.60(\mathrm{~m}, 1 \mathrm{H}), 7.89(\mathrm{~s}, 1 \mathrm{H}), 8.49 \mathrm{ppm}$ (br s, disappeared after treatment with D2O, 1H). IR: $\mathrm{n} 3439$ cm_1.MS (ESI): 398.4 (MHp). C21H19NO3S2 (397.51). 
5.1.4. 5-(Thiophen-3-yl)-3-((3,4,5-trimethoxyphenyl)thio)-1Hindole (6)

Synthesized as 3 starting from 30. Yield 40\%, mp 116e120_C (from ethanol). 1H NMR (DMSO-d6, 400 MHz): d 3.57 (s, $3 \mathrm{H}), 3.58(\mathrm{~s}, 6 \mathrm{H}), 6.44(\mathrm{~s}, 2 \mathrm{H}), 7.49 \mathrm{e} 7.51(\mathrm{~m}, 1 \mathrm{H}), 7.53(\mathrm{~s}, 1 \mathrm{H}), 7.55(\mathrm{~d}, \mathrm{~J} 1 / 41.3 \mathrm{~Hz}, 1 \mathrm{H}), 7.59 \mathrm{e} 7.62(\mathrm{~m}, 1 \mathrm{H}), 7.72 \mathrm{e} 7.74(\mathrm{~m}$, $2 \mathrm{H}), 7.79(\mathrm{~d}, \mathrm{~J} 1 \mathrm{4} 2.4 \mathrm{~Hz}, 1 \mathrm{H}), 11.71 \mathrm{ppm}$ (br s, disappeared after treatment with D2O, 1H). IR: $\mathrm{n} 3448 \mathrm{~cm} \_1$. MS (ESI): 398.5 (MHp). C21H19NO3S2 (397.51).

5.1.5. 6-(Furan-2-yl)-3-((3,4,5-trimethoxyphenyl)thio)-1H-indole (7)

Synthesized as 3 starting from 31. Yield 44\%, mp 164e169_C (from ethanol). 1H NMR (DMSO-d6, 300 MHz): d 3.56 (s, $9 \mathrm{H}), 6.37(\mathrm{~s}, 2 \mathrm{H}), 6.56 \mathrm{e} 6.60(\mathrm{~m}, 1 \mathrm{H}), 6.85(\mathrm{~d}, \mathrm{~J} 1 / 43.2 \mathrm{~Hz}, 1 \mathrm{H}), 7.45(\mathrm{~s}, 2 \mathrm{H}), 7.70 \mathrm{e} 7.74(\mathrm{~m}, 3 \mathrm{H}), 11.74 \mathrm{ppm}$ (br s, disappeared after treatment with D2O, 1H). IR: n 3179 cm_1. MS (ESI): 382.2 (MHp). C21H19NO4S (381.45).

5.1.6. 6-(Furan-3-yl)-3-((3,4,5-trimethoxyphenyl)thio)-1H-indole (9)

Synthesized as 3 starting from 32. Yield 4\%, slurry. 1H NMR (DMSO-d6, $400 \mathrm{MHz}$ ): d 3.57 (s, 9H), 6.39 (s, 2H), $6.90 \mathrm{e} 6.95$ $(\mathrm{m}, 1 \mathrm{H}), 7.34 \mathrm{e} 7.36(\mathrm{~m}, 1 \mathrm{H}), 7.40 \mathrm{e} 7.43(\mathrm{~m}, 1 \mathrm{H}), 7.63(\mathrm{~s}, 1 \mathrm{H}), 7.70 \mathrm{e} 7.73(\mathrm{~m}, 2 \mathrm{H}), 8.14(\mathrm{~s}, 1 \mathrm{H}), 11.66 \mathrm{ppm}$ (br s, disappeared after treatment with D2O, 1H). IR: $n 3401 \mathrm{~cm} \_1$. MS (ESI): 382.2 (MHp). C21H19NO4S (381.45).

5.1.7. 6-(Thiophen-2-yl)-3-((3,4,5-trimethoxyphenyl)thio)-1Hindole (11)

Synthesized as 3 starting from 33. Yield 50\%, mp 158e161_C (from ethanol). 1H NMR (DMSO-d6, 400 MHz): d 3.58 (s, $3 \mathrm{H}), 3.59(\mathrm{~s}, 6 \mathrm{H}), 6.41(\mathrm{~s}, 2 \mathrm{H}), 7.10 \mathrm{e} 7.13(\mathrm{~m}, 1 \mathrm{H}), 7.41 \mathrm{e} 7.43(\mathrm{~m}, 1 \mathrm{H}), 7.44 \mathrm{e} 7.48(\mathrm{~m}, 3 \mathrm{H}), 7.70 \mathrm{e} 7.72(\mathrm{~m}, 1 \mathrm{H}), 7.82(\mathrm{~s}$, 1H), 11.74 ppm (br s, disappeared after treatment with D2O, 1H). IR: $n 3372$ cm_1. MS (ESI): 398.3 (MHp). C21H19NO3S2 (397.51).

\subsubsection{6-(Thiophen-3-yl)-3-((3,4,5-trimethoxyphenyl)thio)-1Hindole (13)}

Synthesized as 3 starting from 39. Yield 30\%, mp 188e190_C (from ethanol). 1H NMR (DMSO-d6, 400 MHz): d 3.58 (s, $3 \mathrm{H}), 3.59(\mathrm{~s}, 6 \mathrm{H}), 6.40(\mathrm{~s}, 2 \mathrm{H}), 7-45-7.47(\mathrm{~m}, 2 \mathrm{H}), 7.52 \mathrm{e} 7.57(\mathrm{~m}, 1 \mathrm{H}), 7.60 \mathrm{e} 7.64(\mathrm{~m}, 1 \mathrm{H}), 7.76(\mathrm{~s}, 1 \mathrm{H}), 7.79(\mathrm{~s}, 1 \mathrm{H}), 7.77 \mathrm{e} 7.81$ (m, 1H), 11.72 ppm (br s, disappeared after treatment with D2O, 1H). IR: n 3376 cm_1. MS (ESI): 398.5 (MHp). C21H19NO3S2 (397.51).

5.1.9. 7-(Furan-2-yl)-3-((3,4,5-trimethoxyphenyl)thio)-1H-indole (15)

Synthesized as 3 starting from 35. Yield 15\%, mp 80e83_C (from ethanol). 1H NMR (DMSO-d6, $400 \mathrm{MHz}$ ): d 3.58 (s, 9H), $6.42(\mathrm{~s}, 2 \mathrm{H}), 6.71 \mathrm{e} 6.75(\mathrm{~m}, 1 \mathrm{H}), 7.16 \mathrm{e} 7.21(\mathrm{~m}, 2 \mathrm{H}), 7.44 \mathrm{e} 7.50(\mathrm{~m}, 1 \mathrm{H}), 7.59 \mathrm{e} 7.63(\mathrm{~m}, 1 \mathrm{H}), 7.80 \mathrm{e} 7.83(\mathrm{~m}, 2 \mathrm{H}), 11.59 \mathrm{ppm}$ (br s, disappeared after treatment with D2O, 1H). IR: n 3351 cm_1. MS (ESI): 382.2 (MHp). C21H19NO4S (381.45).

5.1.10. 7-(Thiophen-2-yl)-3-((3,4,5-trimethoxyphenyl)thio)-1Hindole (19)

Synthesized as 3 starting from 36. Yield 29\%, mp 153e155_C (from ethanol). $1 \mathrm{H}$ NMR (DMSO-d6, $300 \mathrm{MHz}$ ): d 3.56 (s, $3 \mathrm{H}), 3.58(\mathrm{~s}, 6 \mathrm{H}), 6.43(\mathrm{~s}, 2 \mathrm{H}), 7.14(\mathrm{t}, \mathrm{J} 1 / 45.8 \mathrm{~Hz}, 1 \mathrm{H}), 7.24(\mathrm{t}, \mathrm{J} 1 / 43.8 \mathrm{~Hz}, 1 \mathrm{H}), 7.32(\mathrm{~d}, \mathrm{~J}$ 1 $/ 45.5 \mathrm{~Hz}, 1 \mathrm{H}), 7.46(\mathrm{~d}, \mathrm{~J}$ 1 $/ 45.9 \mathrm{~Hz}$, $1 \mathrm{H}), 7.56(\mathrm{~d}, \mathrm{~J} \mathrm{y} / 42.7 \mathrm{~Hz}, 1 \mathrm{H}), 7.64(\mathrm{~d}, \mathrm{~J} 1 / 43.8 \mathrm{~Hz}, 1 \mathrm{H}), 7.76(\mathrm{~s}, 1 \mathrm{H}), 11.57 \mathrm{ppm}$ (br s, disappeared after treatment with D2O, 1H). IR: n $3322 \mathrm{~cm} \_1$. MS (ESI): 398.4 (MHp). C21H19NO3S2 (397.51).

5.1.11. 7-lodo-3-((3,4,5-trimethoxyphenyl)thio)-1H-indole (37)

Synthesized as 3 starting from 26. Yield 27\%, mp 100e103_C (from ethanol). 1H NMR (DMSO-d6, 400 MHz): d 3.56 (s, $3 \mathrm{H}), 3.57(\mathrm{~s}, 6 \mathrm{H}), 6.40(\mathrm{~s}, 2 \mathrm{H}), 6.90(\mathrm{t}, \mathrm{J} 1 / 47.6 \mathrm{~Hz}, 1 \mathrm{H}), 7.46(\mathrm{~d}, \mathrm{~J} 1 / 47.8 \mathrm{~Hz}, 1 \mathrm{H}), 7.58(\mathrm{~d}, \mathrm{~J} \mathrm{y} / 47.4 \mathrm{~Hz}, 1 \mathrm{H}), 7.77(\mathrm{~s}, 1 \mathrm{H}), 11.61$ ppm (br s, disappeared after treatment with D2O, 1H). IR: n $3270 \mathrm{~cm} \_1$.

5.1.12. General procedure for the synthesis of derivatives $8,10,12$ and 14. Example. (6-(Furan-2-yl)-1H-indol-3-yl) (3,4,5-trimethoxyphenyl)methanone (8)

Diethylaluminum chloride ( $120 \mathrm{mg}, 1 \mathrm{mmol} ; 1.0 \mathrm{M}$ in hexanes) was added to a _78_C solution of indole 31 (183 mg, 1 $\mathrm{mmol})$ in dichloromethane $(2.5 \mathrm{~mL})$. The reaction mixture was warmed to _10_C, and 3,4,5-trimethoxybenzoyl chloride $(276 \mathrm{mg}, 1.2 \mathrm{mmol}$ ) was added. The reaction mixture was stirred for $2 \mathrm{~h}$, and the temperature rose to 25 _C. Water and chloroform were added, and the layers were separated. The organic phase was washed with brine, dried and filtered. Evaporation of the solvent gave a residue that was purified by column chromatography (silica gel, $n$-hexane: acetone $1 / 4$ $7: 3$ as eluent) to give 8 (53 mg, yield 14\%), mp 190e195_C (from ethanol). 1H NMR (DMSO-d6, $400 \mathrm{MHz}$ ): $\mathrm{d} 3.75$ (s, 3H), $3.85(\mathrm{~s}, 6 \mathrm{H}), 6.58 \mathrm{e} 6.62(\mathrm{~m}, 1 \mathrm{H}), 6.88 \mathrm{e} 6.92(\mathrm{~m}, 1 \mathrm{H}), 7.10(\mathrm{~s}, 2 \mathrm{H}), 7.61(\mathrm{dd}, \mathrm{J} 1 / 41.4$ and $8.4 \mathrm{~Hz}, 1 \mathrm{H}), 7.74 \mathrm{e} 7.78(\mathrm{~m}, 2 \mathrm{H}), 8.13$ $(\mathrm{s}, 1 \mathrm{H}), 8.20 \mathrm{e} 8.24(\mathrm{~m}, 1 \mathrm{H}), 12.11 \mathrm{ppm}$ (br s, disappeared after treatment with D2O, $1 \mathrm{H}) . \mathrm{IR}: \mathrm{n} 1573$ and $3192 \mathrm{~cm} \_1$. MS (ESI): 378.4 (MHp). C22H19NO5 (377.40).

5.1.13. (6-(Furan-3-yl)-1H-indol-3-yl) (3,4,5-trimethoxyphenyl) methanone (10)

Synthesized as 8 starting from 32. Yield 17\%, mp 180e185_C (from ethanol). 1H NMR (DMSO-d6, 300 MHz): d 3.75 (s, $3 \mathrm{H}), 3.85(\mathrm{~s}, 6 \mathrm{H}), 6.95 \mathrm{e} 6.98(\mathrm{~m}, 1 \mathrm{H}), 7.09(\mathrm{~s}, 2 \mathrm{H}), 7.50 \mathrm{e} 7.55(\mathrm{~m}, 1 \mathrm{H}), 7.66(\mathrm{~s}, 1 \mathrm{H}), 7.75(\mathrm{t}, \mathrm{J} / \mathrm{4} 1.4 \mathrm{~Hz}, 1 \mathrm{H}), 8.07(\mathrm{~s}, 1 \mathrm{H})$, $8.18 \mathrm{e} 8.20(\mathrm{~m}, 2 \mathrm{H}), 12.04 \mathrm{ppm}$ (br s, disappeared after treatment with D2O, 1H). IR: $\mathrm{n} 1576$ and $3238 \mathrm{~cm} \_1$. MS (ESI): 378.4 (MHp). C22H19NO5 (377.40).

5.1.14. (6-(Thiophen-2-yl)-1H-indol-3-yl) (3,4,5-trimethoxyphenyl) methanone (12)

Synthesized as 8 starting from 33. Yield 68\%, mp 200e205_C (from ethanol). $1 \mathrm{H}$ NMR (DMSO-d6, 300 MHz): d 3.75 (s, $3 \mathrm{H}), 3.85(\mathrm{~s}, 6 \mathrm{H}), 7.09(\mathrm{~s}, 2 \mathrm{H}), 7.14 \mathrm{e} 7.20(\mathrm{~m}, 1 \mathrm{H}), 7.45 \mathrm{e} 7.50(\mathrm{~m}, 2 \mathrm{H}), 7.56(\mathrm{dd}, \mathrm{J} \mathrm{1} / 41.5 \mathrm{and} 8.3 \mathrm{~Hz}, 1 \mathrm{H}), 7.73 \mathrm{e} 7.76(\mathrm{~m}, 1 \mathrm{H})$, $8.12(\mathrm{~s}, 1 \mathrm{H}), 8.23$ (d, J $1 / 48.2 \mathrm{~Hz}, 1 \mathrm{H}), 12.04 \mathrm{ppm}$ (br s, disappeared after treatment with D2O, 1H). IR: n 1575 and 3219 cm_1. MS (ESI): 394.4 (MHp). C22H19NO4 S (393.46).

5.1.15. (6-(Thiophen-3-yl)-1H-indol-3-yl) (3,4,5-trimethoxyphenyl) methanone (14) 
Synthesized as 8 starting from 34. Yield 69\%, mp 188e192 _C (fromethanol). 1H NMR (DMSO-d6, 400 MHz): d 3.74 (s, $3 \mathrm{H}), 3.84(\mathrm{~s}, 6 \mathrm{H}), 7.08(\mathrm{~s}, 2 \mathrm{H}), 7.60 \mathrm{e} 7.65(\mathrm{~m}, 3 \mathrm{H}), 7.72 \mathrm{e} 7.76(\mathrm{~m}, 1 \mathrm{H}), 7.82 \mathrm{e} 7.85(\mathrm{~m}, 1 \mathrm{H}), 8.10(\mathrm{~s}, 1 \mathrm{H}), 8.22(\mathrm{~d}, \mathrm{~J} / \mathrm{4} 8.3 \mathrm{~Hz}$, 1H), 12.05 ppm (br s, disappeared after treatment with D2O, 1H). IR: n 1733 and 3113 cm_1. MS (ESI): 394.2 (MHp). C22H19NO4 S (393.46).

5.1.16. General procedure for the synthesis of derivatives 16e18,20e22, 30e32, 35 and 36. (7-(Furan-2-yl)-1H-indol-3yl) (3,4,5-trimethoxyphenyl)methanone (16)

A mixture of derivative 38 (437 mg, $1 \mathrm{mmol}$ ), tris(dibenzylideneacetone) dipalladium(0) (20 mg, $0.022 \mathrm{mmol}), 2$ dicyclohexylphosphino-20,60-dimethoxybiphenyl $(3.2 \mathrm{mg}, 0.008 \mathrm{mmol})$ and potassium phosphate tribasic $(435 \mathrm{mg}, 2.05$ $\mathrm{mmol}$ ) was degassed for $20 \mathrm{~min}$. 1-Butanol (2.2 $\mathrm{mL}$ ) and a solution of 2-furanylboronic acid MIDA ester (2-(furan-2-yl)6-methyl-1,3,6,2-dioxazaborocane-4,8-dione) ( $334 \mathrm{mg}, 1.5 \mathrm{mmol}$ ) in the same solvent ( $4.4 \mathrm{~mL}$ ) were added. The reaction mixture was heated at $100 \_$C for 15 h. After cooling, the mixture was treated dropwise with $1 \mathrm{~N} \mathrm{HCl}$ and extracted with ethyl acetate. The organic layer was washed with brine, dried and filtered. Evaporation of the solvent gave a residue that was purified by column chromatography (silica gel, n-hexane:acetone 1/4 2:1 as eluent) to give 16 (124 mg, yield 33\%), mp 221e224_C (from ethanol). 1H NMR (DMSO-d6, $400 \mathrm{MHz}$ ): d $3.76(\mathrm{~s}, 3 \mathrm{H}), 3.85$ (s, 6H), $6.71 \mathrm{e} 6.75(\mathrm{~m}, 1 \mathrm{H}), 7.12$ (s, 2H), $7.15(\mathrm{~d}, \mathrm{~J} 1 / 43.4 \mathrm{~Hz}, 1 \mathrm{H}), 7.31(\mathrm{t}, \mathrm{J} 1 / 47.7 \mathrm{~Hz}, 1 \mathrm{H}), 7.63(\mathrm{dd}, \mathrm{J} 1 / 41.1$ and $7.5 \mathrm{~Hz}, 1 \mathrm{H}), 7.80 \mathrm{e} 7.85(\mathrm{~m}, 1 \mathrm{H}), 7.98(\mathrm{~s}, 1 \mathrm{H})$, 8.23 (dd, J $1 / 41.1$ and $8.0 \mathrm{~Hz}, 1 \mathrm{H}$ ), 11.85 ppm (br s, disappeared after treatment with D2O, 1H). IR: $\mathrm{n} 1623$ and $3427 \mathrm{~cm} \_1$. MS (ESI): 378.3 (MHp). C22H19NO5 (377.40).

5.1.17. 7-(Furan-3-yl)-3-((3,4,5-trimethoxyphenyl)thio)-1H-indole (17)

Synthesized as 16 from 37 and furan-3-boronic acid pinacol ester. Yield 58\%, mp 156e160_C (fromethanol). 1H NMR (DMSO-d6, $400 \mathrm{MHz}$ ): d $3.57(\mathrm{~s}, 3 \mathrm{H}), 3.58(\mathrm{~s}, 6 \mathrm{H}), 6.42(\mathrm{~s}, 2 \mathrm{H}), 7.04(\mathrm{~m}, 1 \mathrm{H}), 7.15(\mathrm{t}, \mathrm{J} 1 / 47.7 \mathrm{~Hz}, 1 \mathrm{H}), 7.40(\mathrm{~m}, 2 \mathrm{H}), 7.78(\mathrm{~s}$, $1 \mathrm{H}), 7.85\left(\mathrm{t}, \mathrm{J} 1 \frac{1}{4} 1.8 \mathrm{~Hz}, 1 \mathrm{H}\right), 8.31(\mathrm{t}, \mathrm{J} 1 / 41.3 \mathrm{~Hz}, 1 \mathrm{H}), 11.39 \mathrm{ppm}$ (br s, disappeared after treatment with $\left.\mathrm{D} 2 \mathrm{O}, 1 \mathrm{H}\right)$. IR: $n$ $3318 \mathrm{~cm} \_1$. MS (ESI): 382.4 (MHp). C21H19NO4S (381.45).

\subsubsection{8. (7-(Furan-3-yl)-1H-indol-3-yl) (3,4,5-trimethoxyphenyl) methanone (18)}

Synthesized as 16 starting from 38 and furan-3-boronic acid pinacol ester. Yield 46\%, mp 215e220_C (from ethanol). 1H NMR (DMSO-d6, $400 \mathrm{MHz}$ ): d $3.76(\mathrm{~s}, 3 \mathrm{H}), 3.85(\mathrm{~s}, 6 \mathrm{H}), 7.00 \mathrm{e} 7.05(\mathrm{~m}, 1 \mathrm{H}), 7.11(\mathrm{~s}, 2 \mathrm{H}), 7.29(\mathrm{t}, \mathrm{J} / \mathrm{t} / \mathrm{7.9} \mathrm{Hz}, 1 \mathrm{H}), 7.42$ (dd, J $1 / 41.1$ and $7.4 \mathrm{~Hz}, 1 \mathrm{H}$ ), $7.86(\mathrm{t}, \mathrm{J} 1 / 41.8 \mathrm{~Hz}, 1 \mathrm{H}), 7.97(\mathrm{~s}, 1 \mathrm{H}), 8.21(\mathrm{dd}, \mathrm{J} \mathrm{y} / 41.2$ and $8.0 \mathrm{~Hz}, 1 \mathrm{H}), 8.25 \mathrm{e} 8.30(\mathrm{~m}, 1 \mathrm{H})$, 11.76 ppm (br s, disappeared after treatment with D2O, 1H). IR: n 1568 and 3285 cm_1. MS (ESI): 378.4 (MHp). C22H19NO5 (377.40).

5.1.19. (7-(Thiophen-2-yl)-1H-indol-3-yl) (3,4,5-trimethoxyphenyl) methanone (20)

Synthesized as 16 starting from 38 and 2-thienylboronic acid. Yield 6\%, mp 209e211_C (from ethanol). 1H NMR (DMSOd6, $400 \mathrm{MHz}$ ): d $3.76(\mathrm{~s}, 3 \mathrm{H}), 3.85(\mathrm{~s}, 6 \mathrm{H}), 7.11(\mathrm{~s}, 2 \mathrm{H}), 7.28 \mathrm{e} 7.33(\mathrm{~m}, 2 \mathrm{H}), 7.38(\mathrm{dd}, \mathrm{J} 1 / 41.2$ and $7.4 \mathrm{~Hz}, 1 \mathrm{H}), 7.56(\mathrm{dd}, \mathrm{J}$ 1/4 1.2 and $3.6 \mathrm{~Hz}, 1 \mathrm{H}$ ), 7.68 (dd, J 1/4 1.1 and $5.1 \mathrm{~Hz}, 1 \mathrm{H}$ ), $7.95(\mathrm{~s}, 1 \mathrm{H}), 8.25$ (dd, J $1 / 41.2$ and $7.9 \mathrm{~Hz}, 1 \mathrm{H}), 11.94 \mathrm{ppm}(\mathrm{br} \mathrm{s}$, disappeared after treatment with D2O, 1H). IR: $n 1573$ and $3263 \mathrm{~cm} \_1 . M S$ (ESI): 394.4 (MHp). C22H19NO4S (393.46).

5.1.20. 7-(Thiophen-3-yl)-3-((3,4,5-trimethoxyphenyl)thio)-1Hindole (21)

Synthesized as 16 starting from 37 and 3-thienylboronic acid. Yield 75\%, mp 147e150_C (from ethanol). 1H NMR (DMSO-d6, $400 \mathrm{MHz}$ ): d $3.57(\mathrm{~s}, 3 \mathrm{H}), 3.58(\mathrm{~s}, 6 \mathrm{H}), 6.43(\mathrm{~s}, 2 \mathrm{H}), 7.15(\mathrm{t}, \mathrm{J}$ 1/4 $7.8 \mathrm{~Hz}, 1 \mathrm{H}), 7.35$ (dd, J 1/4 $1.1 \mathrm{and} 7.4 \mathrm{~Hz}, 1 \mathrm{H}$ ), $7.44(\mathrm{dd}, \mathrm{J} 1 / 41.1$ and $7.9 \mathrm{~Hz}, 1 \mathrm{H}), 7.54(\mathrm{dd}, \mathrm{J} 1 / 41.4$ and $5.0 \mathrm{~Hz}, 1 \mathrm{H}), 7.70 \mathrm{e} 7.75(\mathrm{~m}, 2 \mathrm{H}), 7.80 \mathrm{e} 7.85(\mathrm{~m}, 1 \mathrm{H}), 11.52 \mathrm{ppm}(\mathrm{br}$ s, disappeared after treatment with D2O, 1H). IR: $n 3327$ cm_1. MS (ESI): 398.4 (MHp). C21H19NO3S2 (397.51).

5.1.21. (7-(Thiophen-3-yl)-1H-indol-3-yl) (3,4,5-trimethoxyphenyl) methanone (22)

Synthesized as 16 starting from 38 and 3-thienylboronic acid. Yield 5\%, slurry. 1H NMR (DMSO-d6, 400 MHz): d 3.76 (s,

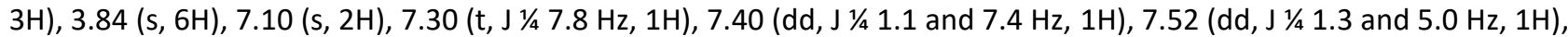
7.70e7.75 (m, 1H), 7.80e7.86 (m, 1H), $7.93(\mathrm{~s}, 1 \mathrm{H}), 8.23(\mathrm{dd}, \mathrm{J} 1 / 41.1$ and $7.8 \mathrm{~Hz}, 1 \mathrm{H}), 11.86 \mathrm{ppm}$ (br s, disappeared after treatment with D2O, 1H). IR: n 1580 and 2925 cm_1. MS (ESI): 394.4 (MHp). C22H19NO4S (393.46).

5.1.22. 5-(Thiophen-3-yl)-1H-indole (30)

Synthesized as 16 starting from 23 and 3-thienylboronic acid. Yield 40\%, mp 80e83 _C (from n-hexane), lit. 80e82 _C [43].

\subsubsection{6-(Furan-2-yl)-1H-indole (31)}

Synthesized as 16 starting from 25 and furan-2-boronic acid MIDA ester. Yield 19\%, mp 90e96 _C (from n-hexane). 1H NMR (DMSO-d6, $400 \mathrm{MHz}$ ): d 6.38e6.42 (m, 1H), 6.50e6.56 (m, 1H), 6.81 (dd, J 1 $1 / 40.8$ and 3.4 Hz, 1H), 7.32e7.36 (m, 2H), $7.55(\mathrm{~d}, \mathrm{~J} \mathrm{y} / 48.3 \mathrm{~Hz}, 1 \mathrm{H}), 7.69 \mathrm{e} 7.71(\mathrm{~m}, 2 \mathrm{H}), 11.17 \mathrm{ppm}$ (br s, disappeared after treatment with D2O, $1 \mathrm{H}) . \mathrm{IR}: \mathrm{n} 3388 \mathrm{~cm} \_1$. 5.1.24. 6-(Furan-3-yl)-1H-indole (32)

Synthesized as 16 starting from 25 and furan-3-boronic acid MIDA ester. Yield 7\%, mp 155e158_C (from n-hexane). 1H NMR (DMSO-d6, $400 \mathrm{MHz}$ ): d 6.37e6.40 (m, 1H), 6.90e6.95 (m, 1H), 7.25 (dd, J 1 1/4 1.6 and 8.2 Hz, 1H), 7.30e7.32 (m, 1H), 7.50e7.53 (m, 2H), $7.77(\mathrm{t}, \mathrm{J} \mathrm{y} / 4.8 \mathrm{~Hz}, 1 \mathrm{H}), 8.08 \mathrm{e} 8.12(\mathrm{~m}, 1 \mathrm{H}), 11.07 \mathrm{ppm}(\mathrm{br} \mathrm{s}$, disappeared after treatment with D2O, 1H). IR: n $3384 \mathrm{~cm} \_1$.

5.1.25. 7-(Furan-2-yl)-1H-indole (35)

Synthesized as 16 starting from 26 and 2-furanylboronic acid MIDA ester. Yield 21\% as an oil [44].

5.1.26. 7-(Thiophen-2-yl)-1H-indole (36) 
Synthesized as 16 starting from 26 and 2-thienylboronic acid. Yield 67\% as an oil [45].

5.1.27. General procedure for the synthesis of derivatives 27 and 29.

\section{Example. 5-(Furan-2-yl)-1H-indole (27)}

A mixture of 24 (243 mg, $1 \mathrm{mmol}$ ), 2-furanboronic acid (223 mg, $2 \mathrm{mmol}$ ), palladium(II) acetate (11 mg, $0.05 \mathrm{mmol})$, tri(o-tolyl) phosphine $(30 \mathrm{mg} \mathrm{g}, 0.1 \mathrm{mmol})$, potassium phosphate tribasic $(745 \mathrm{mg}, 3.5 \mathrm{mmol})$ in ethanol $(10 \mathrm{~mL})$ and toluene $(5 \mathrm{~mL})$ was heated at $80 \_\mathrm{C}$ for $2 \mathrm{~h}$. After cooling, the reaction mixture was diluted with a saturated aqueous solution of sodium hydrogen carbonate and extracted with ethyl acetate. The organic layer was washed with brine, dried and filtered. Evaporation of the solvent gave a residue that was purified by column chromatography (silica gel, $\mathrm{n}$ hexane:ethyl acetate $1 / 4$ 5:1 as eluent) to give 27 (159 mg, yield 87\%), mp 108e110_C (from ethanol), lit. 110e111_C.

\subsubsection{5-(Thiophen-2-yl)-1H-indole (29)}

Synthesized as 27 starting from 2-thienylboronic acid. Yield 73\%, mp 50e51_C (from n-hexane), lit. 53e54 _C [46].

\subsubsection{Synthesis of 5-(furan-2-yl)-1H-indole (28)}

A mixture of 24 ( $243 \mathrm{mg}, 1 \mathrm{mmol}$ ), 3-furanboronic acid pinacol ester ( $253 \mathrm{mg}, 1.3 \mathrm{mmol}$ ), potassium carbonate (179 mg, $1.3 \mathrm{mmol})$ in methylpyrrolidone $(2 \mathrm{~mL})$ and water $(0.15 \mathrm{~mL})$ was degassed for $15 \mathrm{~min}$. Palladium(II) acetate (30 mg, 0.13 $\mathrm{mmol}$ ) was added, and the reaction mixture was placed into the MW cavity (closed vessel mode, Pmax $1 / 4250 \mathrm{psi}$ ). Starting MW irradiation of $200 W$ was used, the temperature being ramped from 25 to 110 C. Once this was reached, taking around $2 \mathrm{~min}$, the mixture was held at this temperature for $15 \mathrm{~min}$ with rapid stirring. The reaction mixture was diluted with water and extracted with ethyl acetate. The organic layer was washed with brine, dried and filtered. Evaporation of the solvent gave a residue that was purified by column chromatography (silica gel, n-hexane:ethyl acetate $1 / 4$ 5:1 as eluent) to give 28 ( $82 \mathrm{mg}$, yield 45\%) mp 89e90_C (from n-hexane), lit. 89e91_C [47].

\subsubsection{Synthesis of 7-iodo-1H-indol-3-yl) (3,4,5-trimethoxyphenyl) methanone (38)}

A mixture of 26 (243 mg, $1 \mathrm{mmol}$ ), 3,4,5-trimethoxybenzoyl chloride (230 $\mathrm{mg}, 1 \mathrm{mmol}$ ) and anhydrous aluminum chloride $(133 \mathrm{mg}, 1 \mathrm{mmol}$ ) in 1,2-dichloroethane $(2 \mathrm{~mL}$ ) was placed into the $\mathrm{MW}$ cavity (closed vessel mode, Pmax $1 / 4$ $250 \mathrm{psi}$ ). A starting MW irradiation of $150 \mathrm{~W}$ was used, the temperature being ramped from 25 to $110 \mathrm{C}$ with rapid stirring. Once $110 \mathrm{C}$ was reached, taking around $1 \mathrm{~min}$, the reaction mixturewas held at this temperature for $2 \mathrm{~min}$, diluted with water, treated with $1 \mathrm{~N} \mathrm{HCl}$ and extracted with chloroform. The organic layer was washed with brine, dried and filtered. Evaporation of the solvent gave a residue that was purified by column chromatography (silica gel, $n$ hexane:ethyl acetate $1 / 4$ 1:1 as eluent) to give 38 (223 mg, yield 51\%, mp 148e153 C (from ethanol). 1H NMR (DMSO-d6, $400 \mathrm{MHz}$ ): d $3.76(\mathrm{~s}, 3 \mathrm{H}), 3.85(\mathrm{~s}, 6 \mathrm{H}), 7.04(\mathrm{t}, \mathrm{J} \mathrm{p} / 4 \mathrm{7}, 6 \mathrm{~Hz}, 1 \mathrm{H}), 7.09(\mathrm{~s}, 2 \mathrm{H}), 7.66(\mathrm{dd}, \mathrm{J} 1 / 41.0$ and 7.6 Hz, 1H), 7,98 (s, 1H), $8.23(\mathrm{dd}, \mathrm{J} 1 / 41.0$ and $7.96 \mathrm{~Hz}, 1 \mathrm{H}), 11.86 \mathrm{ppm}$ (br s, disappeared after treatment with D2O, 1H). IR: n 1577 and 3112 $\mathrm{cm} 1$.

\subsection{Molecular modeling studies}

All molecular modeling studies were performed on a MacPro dual $2.66 \mathrm{GHz}$ Xeon running Ubuntu 14LTS. The tubulin structures were downloaded from the PDB data bank (http://www.rcsb.org/), PDB code: 1SA0 [30], 402A [48], 402B [48], 5CAO [8], 5CB4 [8], 5LYJ [49] and 3HKC [50]. Ligand structures were prepared with Maestro [51]. Proteins were prepared by the protein preparation wizard [52] of Maestro. The docking simulations were performed using Plants [53] and Autodock [54]. Images shown in the manuscript were prepared with Pymol [55].

\subsection{Biological assays}

\subsubsection{Tubulin assembly}

The reaction mixtures contained $0.8 \mathrm{M}$ monosodium glutamate ( $\mathrm{pH} 6.6$ with $\mathrm{HCl}$ in a $2 \mathrm{M}$ stock solution), $10 \mathrm{mM}$ tubulin, $4 \%(\mathrm{v} / \mathrm{v}) \mathrm{DMSO}$, and varying concentrations of drug. Following a $15 \mathrm{~min}$ preincubation at $30 \mathrm{C}$, samples were chilled on ice, GTP to $0.4 \mathrm{mM}$ was added, and turbidity development was followed at $350 \mathrm{~nm}$ in a temperature-controlled recording spectrophotometer for $20 \mathrm{~min}$ at $30 \mathrm{C}$. The extent of reaction was measured. Full experimental details were previously reported [56].

\subsection{2. $[3 \mathrm{H}]$ Colchicine binding assay}

The reaction mixtures contained $1.0 \mathrm{mM}$ tubulin, $5.0 \mathrm{mM} \mathrm{[3H]}$ colchicine, and $5.0 \mathrm{mM}$ inhibitor and were incubated for $10 \mathrm{~min}$ at $37 \mathrm{C}$. Complete details were described previously [57].

\subsubsection{Cell cultures}

Cell lines were obtained from the American Type Culture Collection (ATCC), unless otherwise specified. MCF-7 breast carcinoma, OVCAR-8, and NCl/ADR-RES cells were obtained from the National Cancer Institute drug screening laboratory. U87MG and U343MG cell lines were obtained from the National Institute for Cancer Research of Genoa (Italy) and Cell Lines Service GmbH (Germany), respectively. All cell lines, except as indicated, were grown in Dulbecco's modified Eagle's medium (DMEM) supplemented with $10 \%$ fetal bovine serum (FBS), 20mMHEPES,100 U/mL penicillin, $100 \mathrm{mg} / \mathrm{mL}$ streptomycin, and 1\% L-glutamine; specific requirements include the addition of glucose (4.5 g/L for HT29 and HCT116 cells; $1 \mathrm{~g} / \mathrm{L}$ for HepG2). Cell lines were cultured at $37 \mathrm{C}$ in 5\% CO2/95\% air in a humidified incubator. Treatments were initiated $24 \mathrm{~h}$ after cell seeding using compound 13 or 19 diluted in $0.1 \%$ DMSO, the indicated reference compound, or 0.1\% DMSO vehicle, for $24 \mathrm{e} 72 \mathrm{~h}$ as indicated. T98G and U87MG cells were cultured in RPMI medium and minimum essential medium Eagle, respectively, supplemented with $10 \% \mathrm{FBS}, 2 \mathrm{mM} \mathrm{L-glutamine,} 100 \mathrm{U} / \mathrm{mL}$ 
penicillin, $100 \mathrm{mg} / \mathrm{mL}$ streptomycin and $1 \%$ non-essential amino acids at $37 \mathrm{C}$ in $5 \%$ CO2. The U343MG cells were cultured in minimum essential medium Eagle with $2 \mathrm{mM} \mathrm{L-glutamine} \mathrm{and} \mathrm{Earle's} \mathrm{BSS} \mathrm{adjusted} \mathrm{to} \mathrm{contain} 1.5 \mathrm{mg} / \mathrm{mL}$ sodium bicarbonate and supplemented with $10 \% \mathrm{FBS}, 100 \mathrm{U} / \mathrm{mL}$ penicillin, $100 \mathrm{mg} / \mathrm{mL}$ streptomycin, $1 \%$ non-essential amino acids and $1.0 \mathrm{mM}$ sodium pyruvate at $37 \mathrm{C}$ in $5 \% \mathrm{CO} 2$.

\subsubsection{Cell viability assays}

The methodology for the evaluation of the growth of human MCF-7 breast carcinoma, OVCAR-8, and NCI/ADR-RES cells, obtained from the National Cancer Institute drug screening laboratory, was previously described, except that cells were grown for $96 \mathrm{~h}$ for IC50 determinations [58]. Cell viability of HT29, HCT116 and HepG2 cells was determined using the MTT colorimetric assay. HT29, HepG2 and HCT116 cells were seeded into 24 -well plates to a density of $15 \times 10^{3} / \mathrm{mL}$ in each well. After $24 \mathrm{~h}$ of growth to allow attachment of cells to the wells, test compounds were added at 20-320 nM. After $48 \mathrm{~h}$ of growth and removal of the culture medium, $500 \mathrm{~mL} /$ well of PBS containing $500 \mathrm{mM}$ MTT was added. Cell cultures were further incubated at $37 \mathrm{C}$ for $2 \mathrm{~h}$ in the dark. The solutions were then gently aspirated from each well, and the formazan crystals within the cells were dissolved in propan-2-ol and $0.04 \mathrm{~N} \mathrm{HCl}(200 \mathrm{~mL})$. Optical densities were read at $550 \mathrm{~nm}$ using a Multiskan Spectrum Thermo Electron Corporation reader. The results were expressed as \% relative to vehicle-treated control (0.1\% DMSO), and IC50 values were calculated by nonlinear regression analysis (GraphPad Prism statistics software). The effect of the treatment with compounds 13 or 19 on the T98G, U87MG and U343MG cell lines was estimated using the colorimetric MTS conversion assay, as previously reported.27 After compound incubations, the MTS reagent was added, and the absorbance at $590 \mathrm{~nm}$ was measured by a microplate reader (Wallac, Victor 2, 1420 Multilabel Counter, PerkinElmer). The percentage of proliferating cells after compound exposure was calculated with respect to control cells (100\%). The effect of treatment with compounds 13 or 19 on MV411, THP-1, A-549 and PC3 cell was determined by CellTiter-Fluor ${ }^{\mathrm{TM}}$ (Promega cod. G6082). The cells were seeded in 96 multiwell plates at the indicated densities (MV-4: 5000 cells/50 mL/ well; THP-1: 2000 cells/50 mL/well; A549: 3500 cells/50 mL/well; MDA-MB-231: 3500 cells/50 mL/well; PC-3: 2000 cells/50 mL/well). $24 \mathrm{~h}$ after plating, compound 13 or 19 was added to the cells, and the effect on cell proliferation was determined after $72 \mathrm{~h}$ by the CellTiter-Fluor ${ }^{\mathrm{TM}}$ assay. After adding the reagents to the cell plates, the cells were incubated for $90 \mathrm{~min}$ at $37 \mathrm{C}$, and the fluorescent signal was read by using a TECAN reader. The IC50 and GI50 results were obtained by analysis with GraphPad Prism and Assay Explorer software.

\subsubsection{Statistical analyses}

Graph-Pad Prism 5 software (Graph-Pad Software Inc, San Diego, CA) was used for data analysis and graphic presentations. Statistical analysis was performed by non-linear regression fitting; sigmoidal-dose response curves were performed using the log(inhibitor) vs response analyses. The IC50 value and the maximal efficacy of compounds in inhibiting cell viability (E max) were derived.

\subsubsection{Immunofluorescence and microscopy}

After treatment, HeLa cells directly grown on sterile poly-Llysine (Sigma P4832) coated coverslips were fixed in methanol for $6 \mathrm{~min}$ at $20 \mathrm{C}$ and processed for IF using mouse a-tubulin antibody (Sigma clone B-5-1-2, 1:3000 dilution) followed by FITCconjugated anti-mouse secondary antibody (Jackson Immunoresearch Laboratories). Slides were counterstained with $0.05 \mathrm{mg} / \mathrm{mL}$ DAPI (Sigma) and mounted in Vectashield (Vector). IF-processed cells were examined under an epifluorescence Nikon Eclipse 90i with a QICAM Fast 1394 (QImaging) camera and using the NISElements AR 4.0 software (Nikon). Single cell images were routinely taken using immersion oil 100_objectives with NA 1.3.

\subsubsection{Flow cytometric analysis}

Cell cycle phase distribution was analyzed after incubation with PI (Sigma P4170). All parameters (FS, SS and FL-3) were acquired in a linear amplification scale. Cell aggregates were gated out on the bi-parametric graph FL-3 lin/ratio. Cell death was analyzed using annexin V-FITC (Immunological Sciences, IK-11120). Cell samples were analyzed in a Coulter Epics XL cytofluorometer (Beckman Coulter) equipped with EXPO 32 ADC software. Data from at least 10.000 cells per sample were acquired and processed using Win MDI software.

\subsubsection{RNA extraction, reverse transcription and real-time polymerase chain reaction analysis}

Total cellular RNA was processed, and $1 \mathrm{mg}$ of total RNA was retro-transcribed. The cDNA was used to perform a realtime PCR using p21Cip1/Waf1 and GAPDH primers as indicated in Table 1S, Supporting Information. For p21 Cip1/Waf1 and GAPDH genes, the following protocols were used for the PCRs: initialization at $95 \mathrm{C}$ for $3 \mathrm{~min}$, followed by 40 cycles of 95 _C for $10 \mathrm{~s}, 60 \_\mathrm{C}$ for $10 \mathrm{~s}, 72$ _C for $10 \mathrm{~s}$. The melting program was 95 _C for $5 \mathrm{~s}, 65$ _C for 1 min and 97 _C for 10 $\mathrm{s}$. The rate of temperature increase was 1 _C/s, and fluorescence data were continuously acquired. The relative amounts of target genes were normalized to GAPDH expression by Light Cycler ${ }^{\circledR} 480$ Software version 1.5 (Roche Diagnostics) using the 2DDCt method.

\section{Disclaimer}

The content of this paper is solely the responsibility of the authors and does not necessarily reflect the official views of the National Institutes of Health. 


\section{Acknowledgments}

This research was supported by grants PRIN 2015 n. 2015FCHJ8E (to R.S.), Finanziamenti Ateneo 2016 Sapienza Universit_a di Roma n. RG116154CF287B95 (to G.L.R.), AIRC (Italian Association for Cancer Research, grant n. IG10164 to P.L), and Consiglio Nazionale delle Ricerche Flagship Project InterOmics (to P.L.).

\section{Appendix A. Supplementary data}

Supplementary data associated with this article can be found in the online version, at https://doi.org/10.1016/j.fsi.2016.10.010.

These data include MOL files and InChiKeys of the most importantcompounds described in this article.

\section{References}

[1] E. Nogales, Structural insights into microtubule function, Annu. Rev. Biochem. 69 (2000) 277 e302.

[2] M.A. Jordan, L. Wilson, Microtubules as a target for anticancer drugs, Nat. Rev. Canc. 4 (2004) $253 e 265$.

[3] S. Florian, T.J. Mitchison, Anti-microtubule drugs, Methods Mol. Bioogy 1413 (2016) 403e421.

[4] I. Marzo, J. Naval, Antimitotic drugs in cancer chemotherapy: promises and pitfalls, Biochem. Pharmacol. 86 (2013) $703 e 710$.

[5] E. Mukhtar, V.M. Adhami, H. Mukhtar, Targeting microtubules by natural agents for cancer therapy, Mol. Canc. Therapeut. 13 (2014) $275 \mathrm{e} 284$.

[6] G. Chandrasekaran, P. Tatrai, F. Gergely, Hitting the brakes: targeting microtubule motors in cancer, Br. J. Canc. 113 (2015) 693e698.

[7] B. Bhattacharyya, D. Panda, S. Gupta, S. Banerjee, M, Anti-mitotic activity of colchicine and the structural basis for its interaction with tubulin, Med. Res. Rev. 28 (2008) 155e183.

[8] R.B. Ravelli, B. Gigant, P.A. Curmi, I. Jourdain, S. Lachkar, A. Sobel, M. Knossow, Insight into tubulin regulation from a complex with colchicine and a stathmin-like domain, Nature 428 (2004) 198e202.

[9] C.M. Lin, H.H. Ho, G.R. Pettit, E. Hamel, Antimitotic natural products combretastatin A-4 and combretastatin A-2: studies on the mechanism of their inhibition of the binding of colchicine to tubulin, Biochemistry 28 (1989) 6984e6991. [10] T. Beckers, S. Mahboobi, Natural, semisynthetic and synthetic microtubule inhibitors for cancer therapy, Drugs Future 28 (2003) 767e785.

[11] E. Nogales, M. Whittaker, R.A. Milligan, K.H. Downing, High-resolution model of the microtubule, Cell 96 (1999) $79 \mathrm{e} 88$.

[12] J.H. Nettles, H. Li, B. Cornett, J.M. Krahn, J.P. Snyder, K.H. Downing, The binding mode of epothilone A on alpha, beta-tubulin by electron crystallography, Science 305 (2004) 866e869.

[13] R.M. Buey, E. Calvo, I. Barasoain, O. Pineda, M.C. Edler, R. Matesanz, G. Cerezo, C.D. Vanderwal, B.W. Day, E.J. Sorensen, J.A. Lopez, J.M. Andreu, E. Hamel, J.F. Diaz, Cyclostreptin binds covalently to microtubule pores and luminal taxoid binding sites, Nat. Chem. Biol. 3 (2007) 117e125.

[14] Y.M. Liu, H.L. Chen, H.Y. Lee, J.P. Liou, Tubulin inhibitors: a patent review, Expert Opin. Ther. Pat. 24 (2014) $69 \mathrm{e} 88$. [15] J.J. Field, A. Kanakkanthara, J.H. Miller, Microtubule-targeting agents are clinically successful due to both mitotic and interphase impairment of microtubule function, Bioorg. Med. Chem. 22 (2014) 5050 e5059.

[16] X. Wu, Q. Wang, W. Li, Recent advances in heterocyclic tubulin inhibitors targeting the colchicine binding site, Anti Canc. Agents Med. Chem. 16 (2016) 1325e1338.

[17] D. Cella, A. Peterman, S. Hudgens, K. Webster, M.A. Socinski, M.A, Measuring the side effects of taxane therapy in oncology: the functional assesment of cancer therapy-taxane (FACT-taxane), Cancer 98 (2003) 822e831.

[18] B. Kumar, R. Kumar, I.I. Skvortsova, V. Kumar, Mechanisms of tubulin binding ligands to target cancer cells: updates on their therapeutic potential and clinical trials, Curr. Cancer Drug Targets 17 (2017) 357e375.

[19] D. Fanale, G. Bronte, F. Passiglia, V. Cal_o, M. Castiglia, F. Di Piazza, N. Barraco, A. Cangemi, M.T. Catarella, L. Insalaco, A. Listì, R. Maragliano, D. Massihnia, A. Perez, F. Toia, G. Cicero, V. Bazan, Stabilizing versus Destabilizing the Microtubules: a Double-edge Sword for an Effective Cancer Treatment Option? Analytical Cellular Pathology, Amsterdam, 2015, pp. 690e916, 2015.

[20] Y. Lu, J.J. Chen, M. Xiao, W. Li, D.D. Miller, An overview of tubulin inhibitors that interact with the colchicine binding site, Pharmaceut. Res. 29 (2012) $2943 e 2971$.

[21] C. Kanthou, G.M. Tozer, Microtubule depolymerizing vascular disrupting agents: novel therapeutic agents for oncology and other pathologies, Int. J. Exp. Pathol. 90 (2009) 284e294.

[22] C. Stengel, S.P. Newman, M.P. Leese, B.V. Potter, M.J. Reed, A. Purohit, Class III beta-tubulin expression and in vitro resistance to microtubule targeting agents, Br. J. Canc. 102 (2010) $316 \mathrm{e} 324$.

[23] D. Carta, M.G. Ferlin, An overview on 2-arylquinolin-4(1H)-ones and related structures as tubulin polymerisation inhibitors, Curr. Top. Med. Chem. 14 (2014) 2322 e2345.

[24] R. Patil, S.A. Patil, K.D. Beaman, S.A. Patil, Indole molecules as inhibitors of tubulin polymerization: potential new anticancer agents, an update (2013- 2015), Future Med. Chem. 8 (2016) 1291e1316. 
[25] G. La Regina, R. Bai, W.M. Rensen, E. Di Cesare, A. Coluccia, F. Piscitelli, V. Famiglini, A. Reggio, M. Nalli, S. Pelliccia, E. Da Pozzo, B. Costa, I. Granata, A. Porta, B. Maresca, A. Soriani, M.L. Iannitto, A. Santoni, J. Li, M.M. Cona, F. Chen, Y. $\mathrm{Ni}$, A. Brancale, G. Dondio, S. Vultaggio, M. Varasi, C. Mercurio, C. Martini, E. Hamel, P. Lavia, E. Novellino, R. Silvestri, Towards highly potent cancer agents by modulating the $\mathrm{C}-2$ group of the arylthioindole class of tubulin polymerization inhibitors, J. Med. Chem. 56 (2013) $123 e 149$.

[26] G. La Regina, R. Bai, W.M. Rensen, A. Coluccia, F. Piscitelli, V. Gatti, A. Bolognesi, A. Lavecchia, I. Granata, A. Porta, B. Maresca, A. Soriani, M.L. Iannitto, M. Mariani, A. Santoni, A. Brancale, C. Ferlini, G. Dondio, M. Varasi, C. Mercurio, E. Hamel, P. Lavia, E. Novellino, R. Silvestri, Design and synthesis of 2-heterocyclyl-3-arylthio-1H-indoles as potent tubulin polymerization and cell growth inhibitors with improved metabolic stability, J. Med. Chem. 54 (2011) 8394e8406.

[27] G. La Regina, R. Bai, A. Coluccia, V. Famiglini, S. Pelliccia, S. Passacantilli, C. Mazzoccoli, V. Ruggieri, A. Verrico, A. Miele, L. Monti, M. Nalli, R. Alfonsi, L. Di Marcotullio, A. Gulino, B. Ricci, A. Soriani, A. Santoni, M. Caraglia, S. Porto,

E. Da Pozzo, C. Martini, A. Brancale, L. Marinelli, E. Novellino, S. Vultaggio, M. Varasi, C. Mercurio, C. Bigogno, G. Dondio, E. Hamel, P. Lavia, R. Silvestri, New indole tubulin assembly inhibitors cause stable arrest of mitotic progression, enhanced stimulation of natural killer cell cytotoxic activity, and repression of Hedgehog-dependent cancer, J. Med. Chem. 58 (2015)

5789e5807.

[28] G. La Regina, V. Gatti, V. Famiglini, F. Piscitelli, R. Silvestri, Venting-whileheating microwave-assisted synthesis of 3arylthioindoles, ACS Comb. Sci. 14 (2012) $258 \mathrm{e} 262$.

[29] G. De Martino, G. La Regina, A. Coluccia, M.C. Edler, M.C. Barbera, A. Brancale, E. Wilcox, E. Hamel, M. Artico, R. Silvestri, Arylthioindoles, potent Inhibitors of tubulin polymerization, J. Med. Chem. 47 (2004) 6120e6123.

[30] A. Coluccia, D. Sabbadin, A. Brancale, Molecular modelling studies on arylthioindoles as potent inhibitors of tubulin polymerization, Eur. J. Med. Chem. 46 (2011) 3519e3525.

[31] Y. Wang, H. Zhang, B. Gigant, Y. Yu, Y. Wu, X. Chen, Q. Lai, Z. Yang, Q. Chen, J. Yang, Structures of a diverse set of colchicine binding site inhibitors in complex with tubulin provide a rationale for drug discovery, FEBS J. 283 (2016) $102 \mathrm{e} 111$.

[32] T. Simonart, G. Andrei, D. Parent, J.P. Van Vooren, E. De Clercq, R. Snoeck, In vitro sensitivity of Kaposi's sarcoma cells to various chemotherapeutic agents including acyclic nucleoside phosphonates, Antiviral Chem. Chemother. 10 (1999) $129 \mathrm{e} 134$.

[33] H.H. Ryu, S. Jung, T.Y. Jung, K.S. Moon, I.Y. Kim, Y.L. Jeong, S.G. Jin, J. Pei, M. Wen, W.Y. Jang, Role of metallothionein $1 \mathrm{E}$ in the migration and invasion of human glioma cell lines, Int. J. Oncol. 41 (2012) 1305e1313.

[34] C.A. Lipinski, F. Lombardo, B.W. Dominy, P.J. Feeney, Experimental and computational approaches to estimate solubility and permeability in drug discovery and development settings, Adv. Drug Deliv. Rev. 46 (1997) $3 e 26$.

[35] D.F. Veber, S.R. Johnson, H.Y. Cheng, B.R. Smith, K.W. Ward, K.D. Kopple, Molecular properties that influence the oral bioavailability of drug candidates, J. Med. Chem. 45 (2002) 2615e2623.

[36] D. Lagorce, O. Sperandio, J.B. Baell, M.A. Miteva, B.O. Villoutreix, FAF-Drugs3: a web server for compound property calculation and chemical library design, Nucleic Acids Res. 43 (2015) W200eW207 (Web Server issue).

[37] J.D. Hughes, J. Blagg, D.A. Price, S. Bailey, G.A. Decrescenzo, R.V. Devraj, E. Ellsworth, Y.M. Fobian, M.E. Gibbs, R.W. Gilles, N. Greene, E. Huang, T. Krieger-Burke, J. Loesel, T. Wager, L. Whiteley, Y. Zhang, Physiochemical drug properties associated with in vivo toxicological outcomes, Bioorg. Med. Chem. Lett 18 (2008) 4872 e4875.

[38] T. Cheng, Y. Zhao, X. Li, F. Lin, Y. Xu, X. Zhang, Y. Li, R. Wang, L. Lai, Computation of octanol-water partition coefficients by guiding an additive model with knowledge, J. Chem. Inf. Model. 47 (2007) 2140e2148.

[39] J.S. Delaney, ESOL: estimating aqueous solubility directly from molecular structure, J. Chem. Inf. Comput. Sci. 44 (2004) 1000e1005.

[40] H. Pajouhesh, G.R. Lenz, Medicinal chemical properties of successful central nervous system drugs, NeuroRx 2 (2005) 541e553.

[41] S.K. Carlsson, S.P. Brothers, C. Wahlestedt, Emerging treatment strategies for glioblastoma multiforme, EMBO Mol. Med. 6 (2014) 1359e1370.

[42] J. Cmielov_a, M. Rez_a_cov_a, p21Cip1/Waf1 protein and its function based on a subcellular localization, J. Cell. Biochem. 112 (2011) 3502e3506.

[43] K. Billingsley, S.L. Buchwald, Highly efficient monophosphine-based catalyst for the palladium-catalyzed SuzukiMiyaura reaction of heteroaryl halides and heteroaryl boronic acids and esters, J. Am. Chem. Soc. 129 (2007) 3358 e3366. [44] M.L. Read, A. Krapp, P.O. Miranda, L.-L. Gundersen, Synthesis of complex fused polycyclic heterocycles utilizing IMDAF reactions of allylamino- or allyloxyfuryl( hetero)arenes, Tetrahedron 68 (2012) 1869e1885.

[45] L. Wiesemann, M. Kleih, H.A. Mayer, Aromatic aza-bicyclic compounds comprising $\mathrm{Cu}, \mathrm{Ag}, \mathrm{Au}, \mathrm{Zn}, \mathrm{Al}$ for use in electroluminescent devices, PCT Int. Appl. (September 13, 2013). WO 2014067598 A1.

[46] T. Tsuchimoto, Y. Iketani, M. Sekine, Zinc-catalyzed dehydrogenative N-silylation of indoles with hydrosilanes, Chem. Eur J. 18 (2012) 9500e9504. 
[47] M.A. Oberli, S.L. Buchwald, A general method for Suzuki-Miyaura coupling reactions using lithium triisopropyl borates, Org. Lett. 14 (2012) $4606 \mathrm{e} 4609$.

[48] A.E. Prota, F. Danel, F. Bachmann, K. Bargsten, R.M. Buey, J. Pohlmann, S. Reinelt, H. Lane, M.O. Steinmetz, The novel microtubule-destabilizing drug BAL27862 binds to the colchicine site of tubulin with distinct effects on microtubule organization, J. Mol. Biol. 426 (2014) 1848e1860.

[49] R. Gaspari, A.E. Prota, K. Bargsten, A. Cavalli, M.O. Steinmetz, Structural basis of cis- and trans-combretastatin binding to tubulin, Inside Chem. 2 (2017) 102e113.

[50] A. Dorleans, B. Gigant, R.B.G. Ravelli, P. Mailliet, V. Mikol, M. Knossow, Variations in the colchicine-binding domain provide insight into the structural switch of tubulin, Proc. Natl. Acad. Sci. U.S.A. 106 (2009) 13775e13779.

[51] Schr€odinger Release 2017-3, Maestro, Schr€odinger, LLC, New York, NY, 2017.

[52] G.M. Sastry, M. Adzhigirey, T. Day, R. Annabhimoju, W. Sherman, Protein and ligand preparation: parameters, protocols, and influence on virtual screening enrichments, J. Comput. Aided Mol. Des. 27 (2013) 221 e234.

[53] O. Korb, T. Stutzle, T.R. Exner, Plants: application of ant colony optimization to structure-based drug design, in: M. Dorigo, L.M. Gambardella, M. Birattari, A. Martinoli, R. Poli, T. Stutzle (Eds.), Ant Colony Optimization and Swarm Intelligence, Proceedings of the 5th International Workshop, Ants, Springer, Berlin, 2006, pp. 247e258. Lecture Notes in Computer Science, Series 4150.

[54] G.M. Morris, R. Huey, W. Lindstrom, M.F. Sanner, R.K. Belew, D.S. Goodsell, A.J. Olson, Autodock4 and AutoDockTools4: automated docking with selective receptor flexiblity, J. Comput. Chem. 16 (2009) 2785 e2791.

[55] The Pymol Molecular Graphics System, Version 2.0 Schr€odinger, (LLC).

[56] E. Hamel, E, Evaluation of antimitotic agents by quantitative comparisons of their effects on the polymerization of purified tubulin, Cell Biochem. Biophys. 38 (2003) 1 e21.

[57] P. Verdier-Pinard, J.-Y. Lai, H.-D. Yoo, J. Yu, B. Marquez, D.G. Nagle, M. Nambu, J.D. White, J.R. Falck, W.H. Gerwick, B.W. Day, E. Hamel, Structure-activity analysis of the interaction of curacin A, the potent colchicine site antimitotic agent, with tubulin and effects of analogs on the growth of MCF-7 breast cancer cells, Mol. Pharmacol. 35 (1998) 62 e76. [58] S. Ruan, M.F. Okcu, R.C. Pong, M. Andreeff, V. Levin, J.T. Hsieh, W. Zhang, W, Attenuation of WAF1/Cip1 expression by an antisense adenovirus expression vector sensitizes glioblastoma cells to apoptosis induced by chemotherapeutic agents 1,3-bis(2-chloroethyl)-1-nitrosourea and cisplatin, Clin. Canc. Res. 5 (1999) 197e202.

Figures and Tables<smiles>COc1cc(Sc2c(-c3cccs3)[nH]c3ccccc23)cc(OC)c1OC</smiles>

1

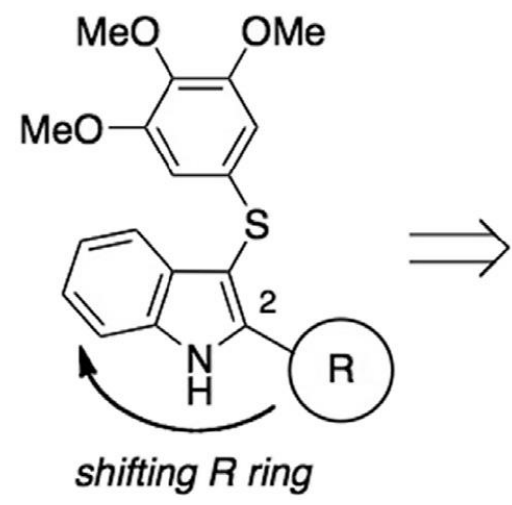<smiles>COc1cc(Sc2c(-c3ccccc3)[nH]c3cc(Cl)ccc23)cc(OC)c1OC</smiles>

2

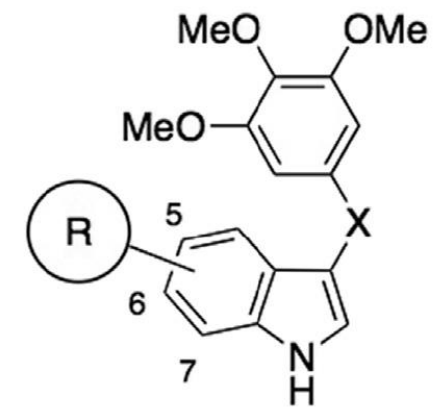

3-22 (see Table 1)

Chart 1. Structures of compounds $1-22$ 
Table 1

Inhibition of tubulin polymerization, growth of MCF-7 human breast carcinoma cells, and colchicine binding by compounds 3-22.a.2

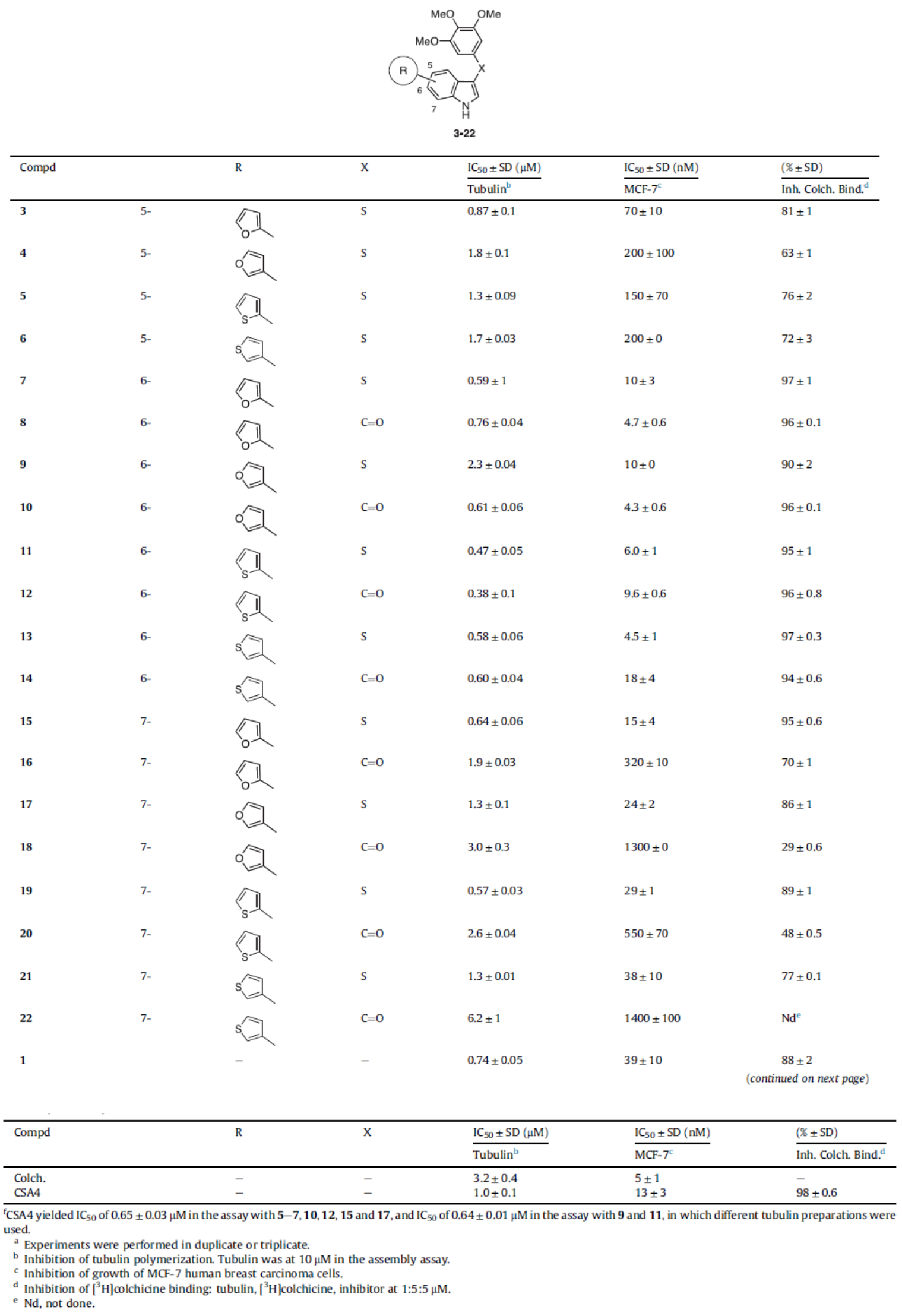




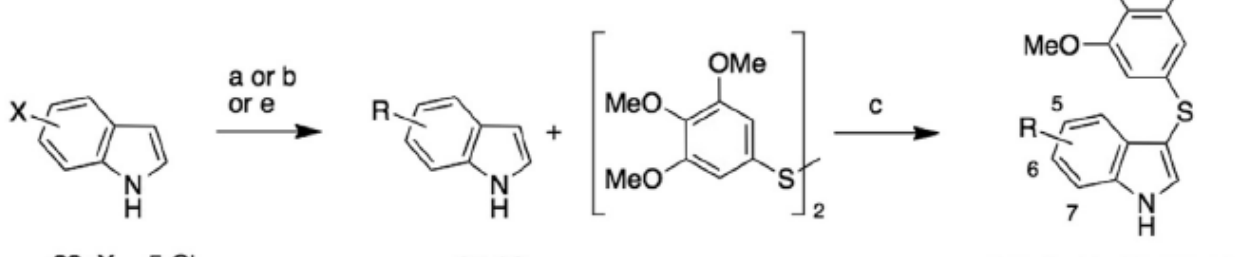

23: $X=5-C l$
24: $X=5-I$
25: $X=6-B r$
26: $X=7-\mid$
27: $R=5$-furan-2-yl
28: $R=5$-furan-3-yl
29: $R=5$-thiophen-2-yl
30: $R=5$-thiophen-3-yl
31: $R=6$-furan-2-yl
32: $R=6$-furan-3-yl
33: $R=6$-thiophen-2-yl
34: $R=6$-thiophen-3-yl
35: $R=7$-furan-2-yl
36: $R=7$-thiophen-2-yl.

27-36

$3-7,9,11,13,15,19$
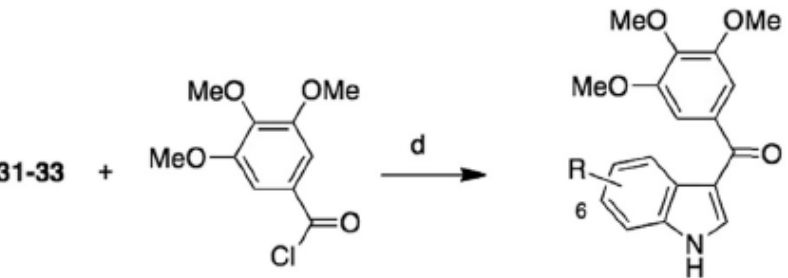

$8,10,12,14$<smiles>COc1cc(Sc2c[nH]c3c(I)c[nH]c23)cc(OC)c1OC</smiles>

37

17,21<smiles>COc1cc(C(=O)Cl)cc(OC)c1OC</smiles><smiles>COc1cc(C(=O)c2c[nH]c3c(I)cccc23)cc(OC)c1OC</smiles><smiles>[2H]c1cccc2c(C(=O)c3cc(OC)c(OC)c(OC)c3)c[nH]c12</smiles>

$16,18,20,22$

Scheme 1. Synthesis of Compounds $3 \mathrm{e} 22$ and 27e37. Reagents and reaction conditions: (a) (27, 29) boronic derivate, $\mathrm{Pd}(\mathrm{OAc}) 2, \mathrm{P}(\mathrm{o}-\mathrm{tol}) 3, \mathrm{~K} 3 \mathrm{PO}$, EtOH/PhMe, 80 C, 2 h, 73e87\%; (b) (28) 3-furanboronic acid pinacol ester, Pd(OAc)2, K2CO3, methylpyrrolidone/water, closed vessel, 200W, 110_C, $15 \mathrm{~min}, 45 \%$; (c) $(3 \mathrm{e} 7,9,11,13,15,19$ and 37) bis(3,4,5-trimethoxyphenyl)disulfide, NaH, anhydrous DMF, closed vessel, 120W, 130_C, 2 min, 5e50\%; (d) $(8,10,12,14)$ (i) diethylaluminum chloride, $\mathrm{CH} 2 \mathrm{Cl} 2,-78$ _C, Ar stream; (ii) 3,4,5-trimethoxybenzoyl chloride, _10_C/25_C within 2 h, 14e69\%; (e) (16e18, 20e22, 30e32, 35 and 36) boronic derivative, Pd2dba3, SPhos, K3PO4, BuOH, 100 _, 15 h, 5e75\%; (f) (38) 3,4,5-trimethoxybenzoyl chloride, $\mathrm{CH} 2 \mathrm{Cl} 2$, anhydrous $\mathrm{AlCl} 3$, closed vessel, 150W, $110 \_\mathrm{C}, 2 \mathrm{~min}, 51 \%$.

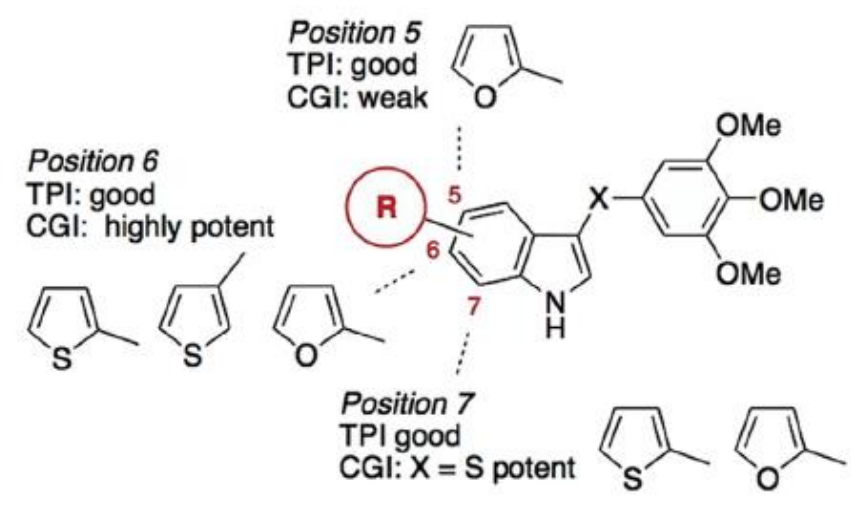

Chart 2. SAR summary for tubulin polymerization inhibition (TPI) and MCF-7 cell growth inhibition (CGI) by compounds 3-22. 


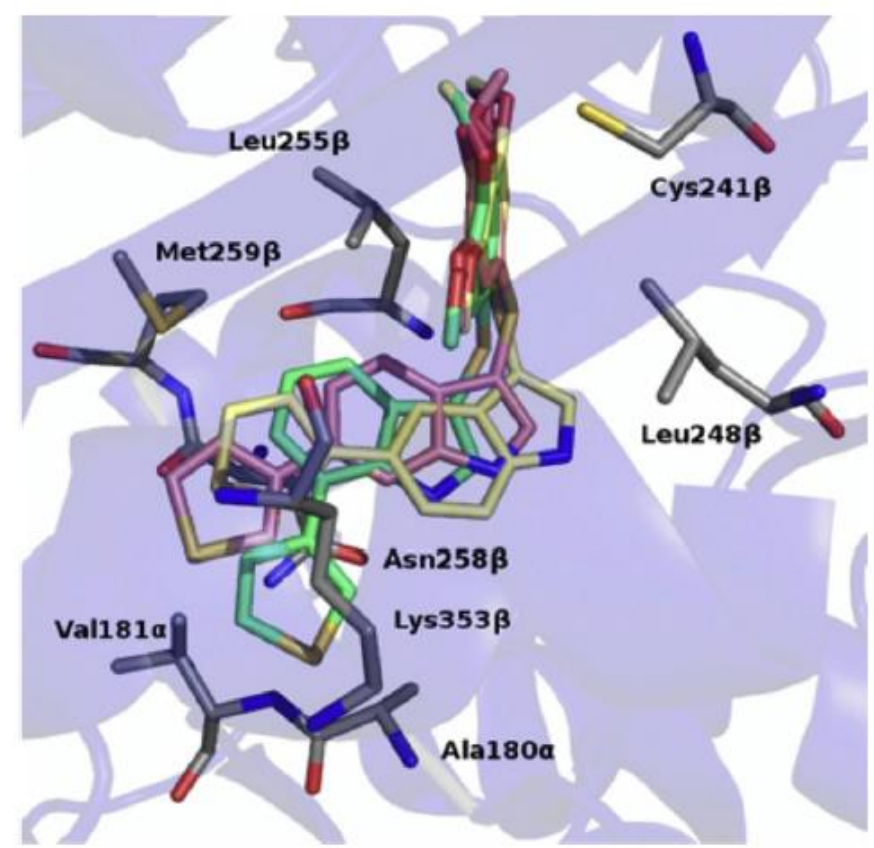

Fig. 1. Plants proposed binding mode for derivatives 6 (yellow), 19 (pink) and 21 (green) into the 1SA0 tubulin structure. Residues involved in interactions are shown as stick diagrams. The tubulin polypeptide chains are shown as ribbon cartoons. (For interpretation of the references to color in this figure legend, the reader is referred to the Web version of this article.)

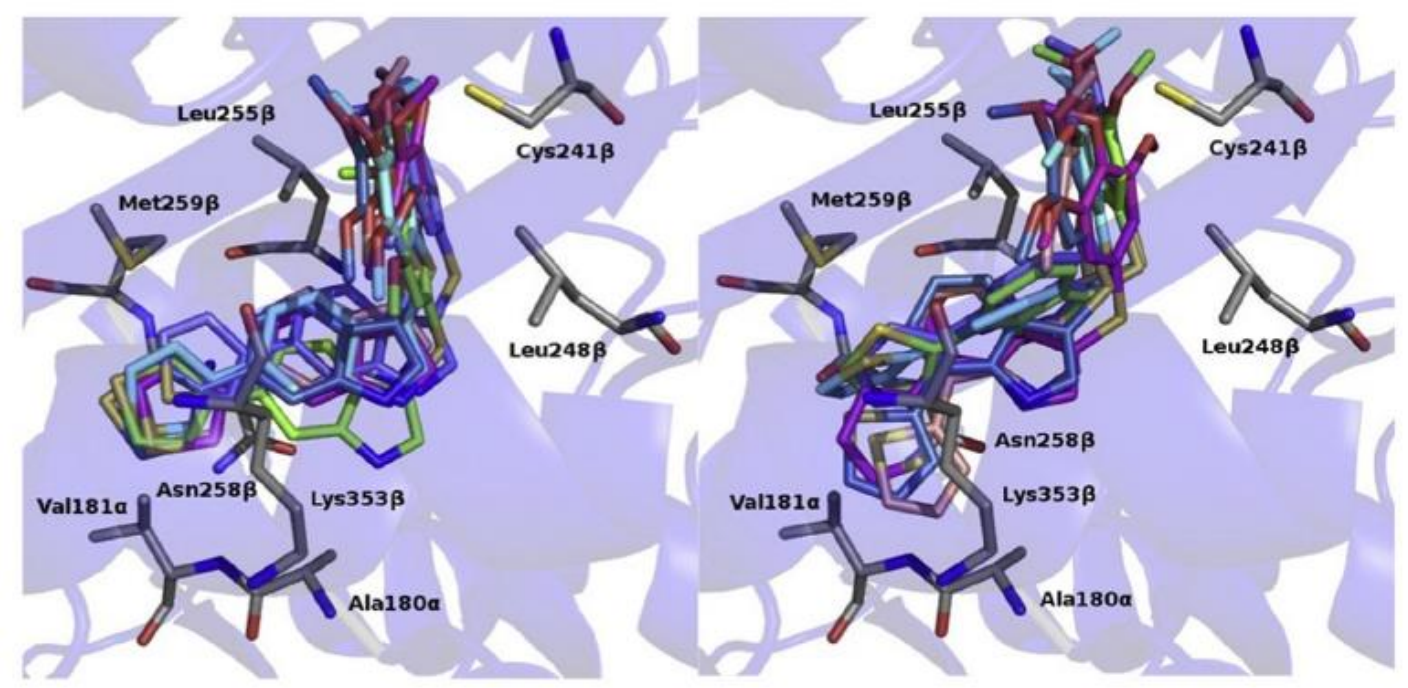

Fig. 2. Plants proposed binding mode for derivatives 13 (left panel) and 19 (right panel) for the studied tubulin structures: $1 \mathrm{SAO}$ (green), $3 \mathrm{HKC}$ (cyan), 4O2B (magenta), 5CA0 (purple), 5CB4 (sky blue), 5LYJ (pink). Residues involved in interactions are shown as stick diagrams. The tubulin polypeptide chains are shown as ribbon cartoons. Residues and cartoon are from 1SA0. (For interpretation of the references to color in this figure legend, the reader is referred to the Web version of this article.)

\section{Table 2}

Growth Inhibition of HT29, HCT116, HepG2, T98G, U87MG, U343G, MV4-11, THP-1, A-549 and PC3 cells by 13 and 19.a,b

\begin{tabular}{lllllllllll}
\hline Compd & \multicolumn{1}{l}{$\mathrm{I}_{50} \pm \mathrm{SD}(\mathrm{nM})$} & & & & & \\
\cline { 2 - 10 } & HT29 & HCT116 & HepG2 & T98G & U87 MG & U343G & MV4-11 & THP-1 & A-549 & PC3 \\
\hline $\mathbf{1 3}$ & $10 \pm 1.4$ & $21 \pm 1.5$ & $20 \pm 1.1$ & $26 \pm 3.6$ & $16 \pm 2.0$ & $31 \pm 4.3$ & $6 \pm 2.0$ & $2 \pm 1$ & $2 \pm 0.1$ & $3.5 \pm 2$ \\
19 & $69 \pm 1.4$ & $70 \pm 1.7$ & $80 \pm 1.2$ & $211 \pm 20$ & $96 \pm 14$ & $154 \pm 6.9$ & $70 \pm 16$ & $20 \pm 2$ & $58 \pm 1$ & $39 \pm 4$ \\
\hline
\end{tabular}

${ }^{a}$ Cytotoxic concentrations for the indicated cell lines; cytostatic concentrations for A-549 and PC3 cells.

b Incubation time was 48 h; for U343G, MV4-11 and PC3 cells, incubation was $72 \mathrm{~h}$. 
Table 3

Growth Inhibition of the OVCAR-8 and NCI/ADR-RES cells by compounds 13 and 19 and reference compounds CSA4, VRB, VLB and PTX.a

\begin{tabular}{|c|c|c|c|}
\hline \multirow[t]{2}{*}{ Compd } & \multicolumn{3}{|c|}{$\mathrm{IC}_{50} \pm \mathrm{SD}(\mathrm{nM})$} \\
\hline & OVCAR-8 & NCl/ADR_RES & MCF-7 \\
\hline 13 & $9.3 \pm 2$ & $7.5 \pm 2$ & $6.7 \pm 2$ \\
\hline 19 & $58 \pm 8$ & $34 \pm 8$ & $55 \pm 7$ \\
\hline CSA4 & $4.0 \pm 0^{\mathrm{b}}$ & $3.5 \pm 0.7$ & $5.0 \pm 1$ \\
\hline PTX & $4.0 \pm 1$ & $3100 \pm 600$ & $5.5 \pm 0.7$ \\
\hline VRB & $300 \pm 0$ & $5000 \pm 1000$ & $\mathrm{Nd}^{\mathrm{c}}$ \\
\hline VBL & $15 \pm 7$ & $200 \pm 0$ & Nd \\
\hline
\end{tabular}

${ }^{a}$ Inhibition of growth of the indicated cell lines.

b Same value obtained in all experiments.

${ }^{c} \mathrm{Nd}$, not done.
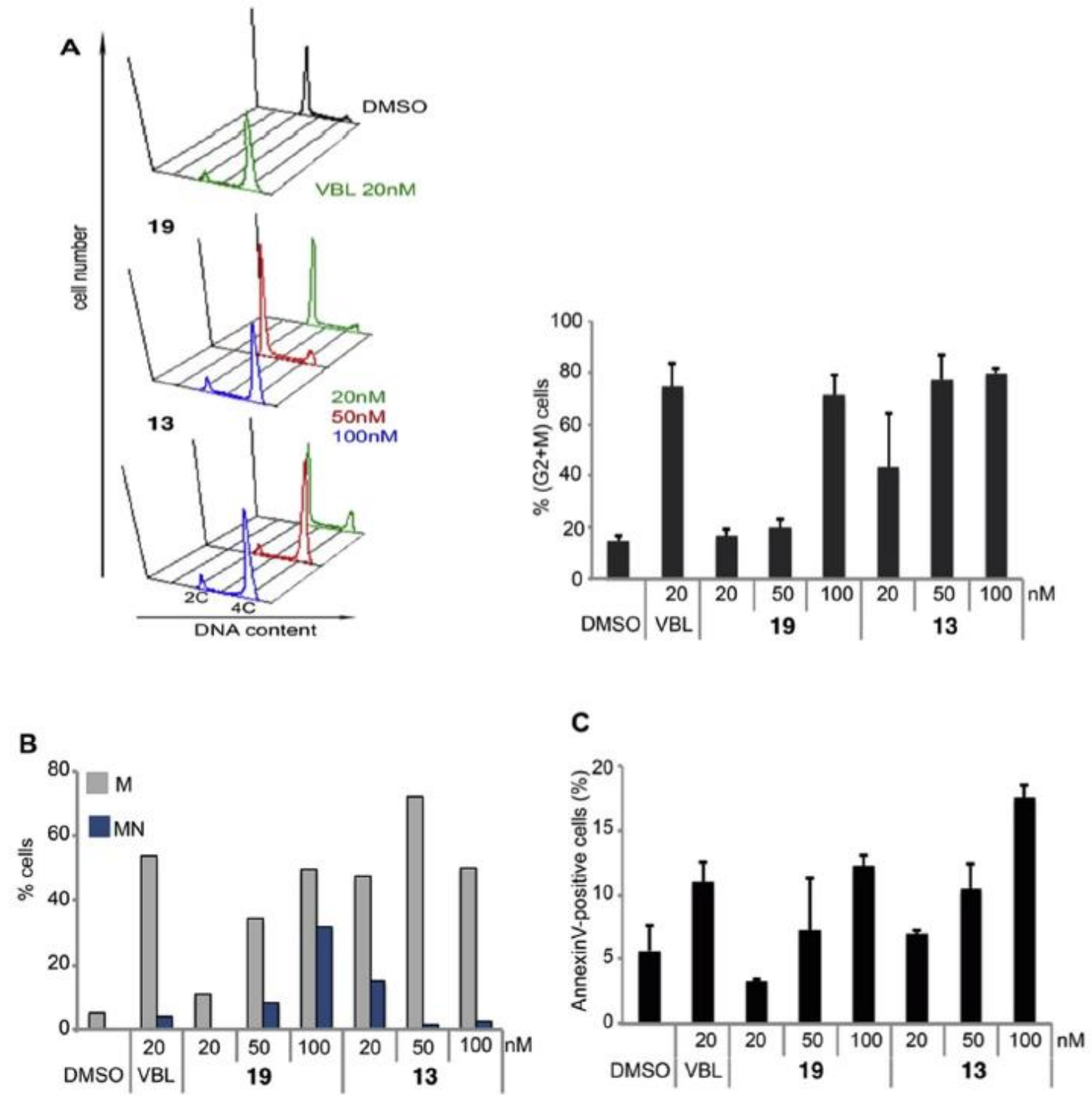

Fig. 3. FACS analysis of the cell cycle and cell death in HeLa cell populations treated with 13 or 19 . Panel A left: distribution of cells after a $24 \mathrm{~h}$ treatment with 13 or 19 or VBL (DMSO as control). The DNA content indicates the cell cycle phase (2C: G1; 4C: G2pM; intermediate values between $2 \mathrm{C}$ and $4 \mathrm{C}$ : $\mathrm{S}$ phase). The panel shows a representative analysis of the cell cycle phase distribution, arranged according to the compound concentration (on the $z$ axis). In the lower two diagrams, $20 \mathrm{nM}$, green curves; $50 \mathrm{nM}$, red curves; $100 \mathrm{nM}$; blue curves. The profiles illustrate that 19 only induces $4 \mathrm{C}$ cell accumulation when used at $100 \mathrm{nM}$, whereas 13 is fully effective at $50 \mathrm{nM}$. Panel A right: the histograms show the mean \% values \pm SD of $4 \mathrm{C}$ cells (G2pM phases) counted in 3 independent experiments. Panel B: the graph represents the frequency of mitotic figures (grey bars) and multinucleated cells (blue bars) in cell populations treated for $24 \mathrm{~h}$ under the indicated conditions, processed for immunofluorescence and scored by microscopy. Between 300 and 650 cells were counted for each condition in 2 independent experiments. Panel C: FACS analysis of cell populations 
treated for $24 \mathrm{~h}$ under the indicated conditions and stained with annexin V. Mean \pm SD values were calculated from three experiments. (For interpretation of the references to color in this figure legend, the reader is referred to the Web version of this article.)

A

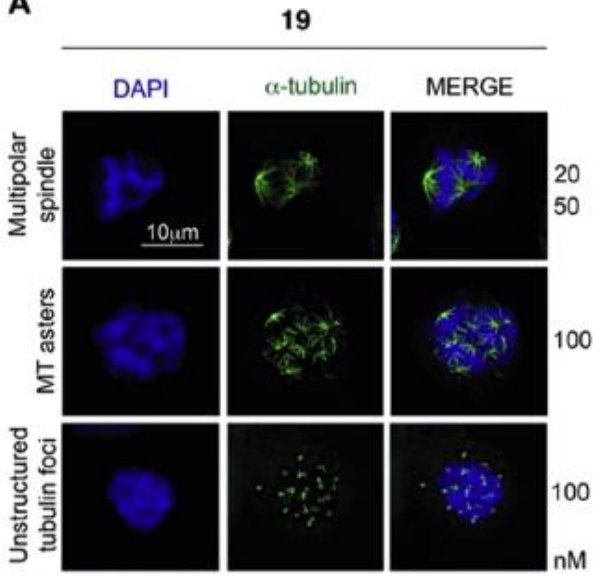

B

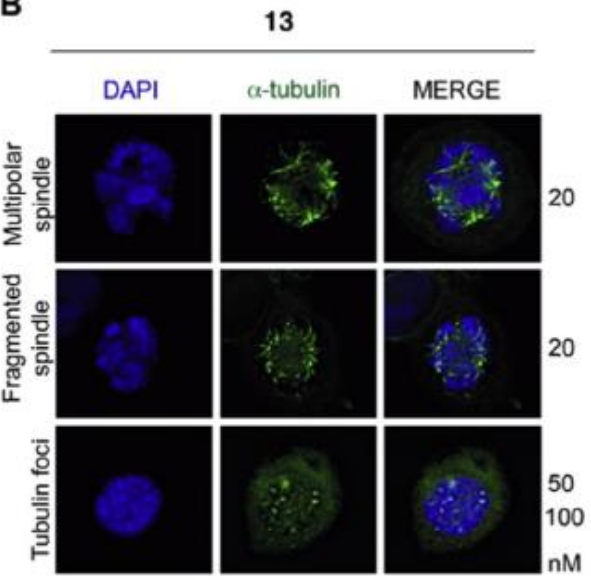

\section{C}

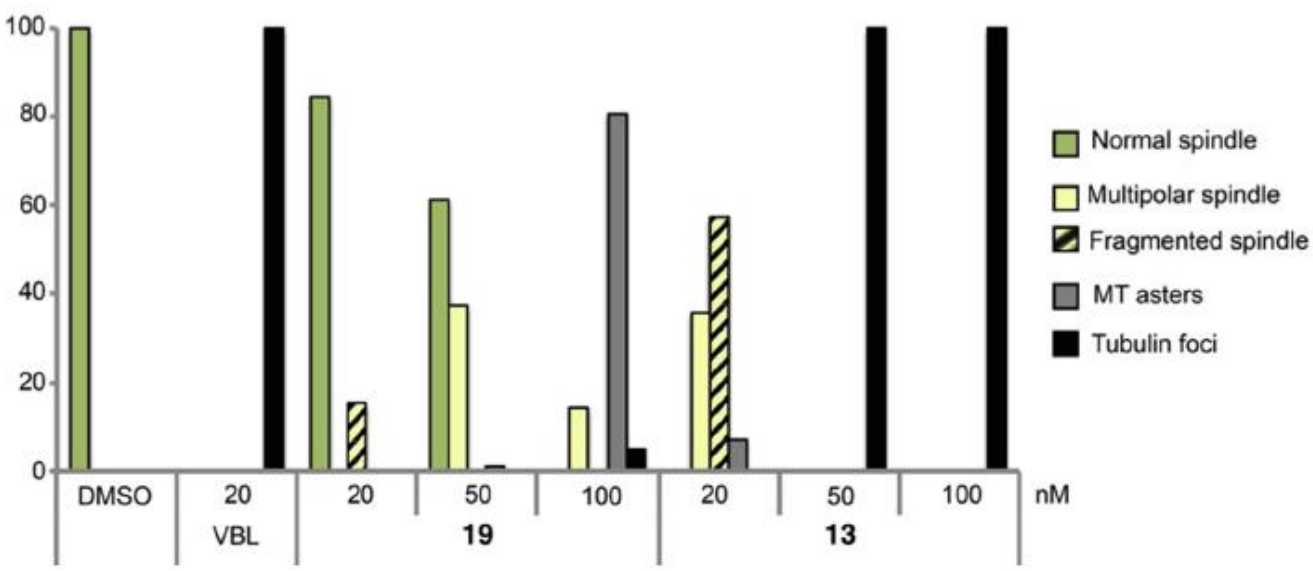

Fig. 4. IF analysis of mitotic cells in HeLa cultures treated with 13 or 19. Panel A: representative phenotypes induced by 19: all cells were arrested in prometaphase (compare the configuration of chromosomes in DAPI panels); the green channel shows the extent of inhibition of tubulin polymerization at increasing compound concentrations (indicated at the right of the panels in nM). Panel B: representative phenotypes induced by 13: the DAPI panels show the prometaphase configuration of chromosomes; the green channel shows the extent of inhibition of tubulin polymerization at the indicated concentrations. Panel C: Distribution of tubulin cytological phenotypes under the indicated conditions. The histograms represent the frequency of the indicated phenotypes ( $n, 100$ to 250 prometaphase-arrested cells per condition). (For interpretation of the references to color in this figure legend, the reader is referred to the Web version of this article.)

Table 4

Physico-chemical profiles of compounds 13 and 19

\begin{tabular}{|c|c|c|c|c|c|c|c|c|}
\hline Compd & $\log P^{a}$ & $\mathrm{MW}^{\mathrm{b}}$ & $\log S w^{c}$ & tPSA $^{\mathrm{d}}$ & HBA-HBD ${ }^{e}$ & RoF viol $^{f}$ & Veber $^{g}$ & $3 / 75^{\mathrm{h}}$ \\
\hline 13 & 5.34 & 397.51 & -5.58 & 97.02 & 5 & 1 & good & warning \\
\hline 19 & 5.37 & 397.51 & -5.60 & 97.02 & 5 & 1 & good & warning \\
\hline $\begin{array}{l}\text { a Logarit } \\
\text { b Molech } \\
\text { c LogSw } \\
\text { d Molec } \\
\text { e Numbe } \\
\text { f Violati } \\
\text { g Veber' } \\
\text { h } 3 / 75 \text { r }\end{array}$ & $\begin{array}{l}\text { partitio } \\
\text { th. } \\
\text { ts the log } \\
\text { surface } \\
\text { accepto } \\
\text { rule of fi } \\
\text { ching [3 } \\
\text { ing [36]. }\end{array}$ & $\begin{array}{l}\text { nt betwe } \\
\text { compoun } \\
\text { parameter }\end{array}$ & $\begin{array}{l}\text { nol and wa } \\
\text { solubility c } \\
\text { shown to }\end{array}$ & $\begin{array}{l}\text { uted by } \\
\text { by the } \mathrm{E} \\
\text { with hu }\end{array}$ & $\begin{array}{l}\text { ethod [38]. } \\
\text { tinal absorpti }\end{array}$ & {$[40]$.} & & \\
\hline
\end{tabular}




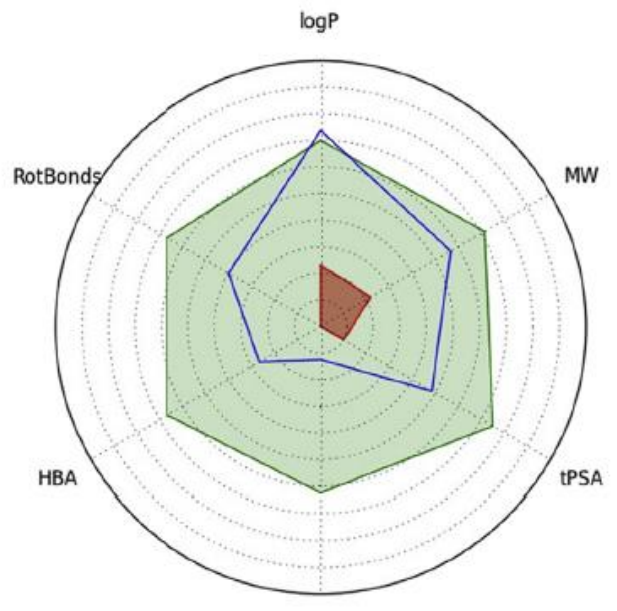

HBD

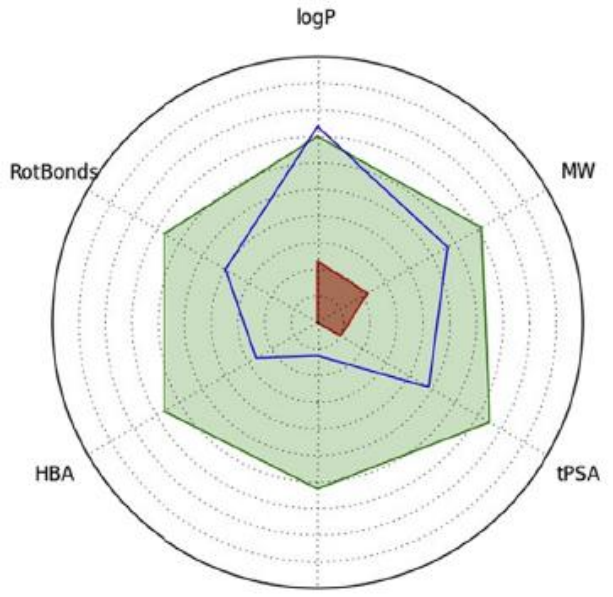

HBD
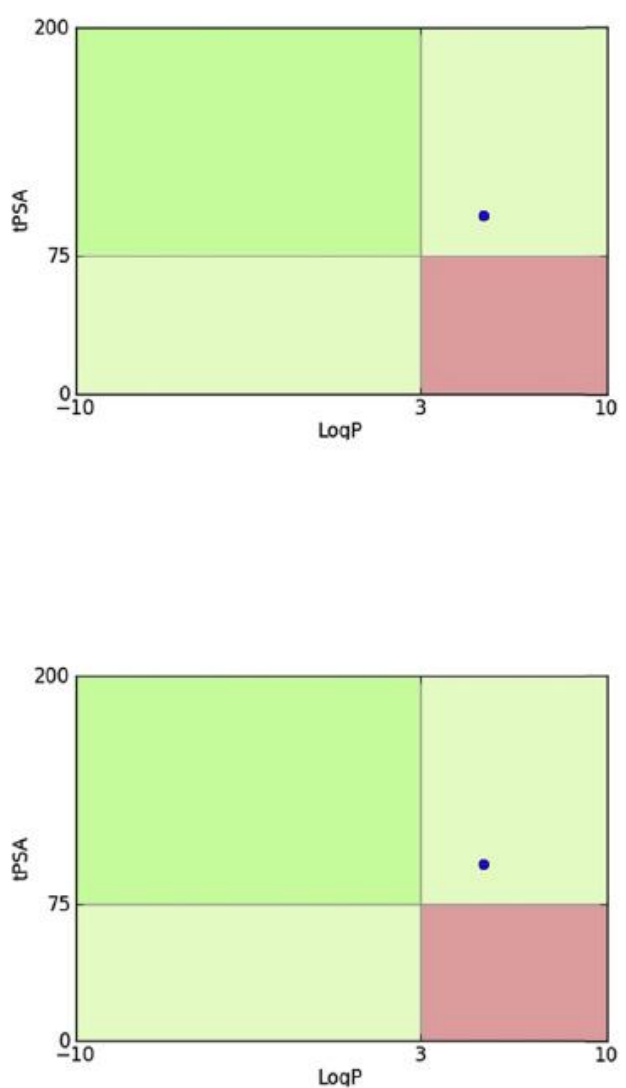

Fig. 5. Physico-chemical properties. Left. Compounds 13 and 19 (blue lines) are compared with the optimal light green area defined by Lipinski's ruleof-five and Veber's rule. The computations includes $\log \mathrm{P}$, molecular weight (MW), topological polar surface area (tPSA), rotatable bonds (RotB), $\mathrm{H}$ bond acceptors and donors (HBA, HBD. The red area indicates low oral bioavailability. Estimated physico-chemical profiles of compounds 13 and 19 fall within the light green area. Right. Likelihood of in vivo toxicity according 3/75 rule [37]. tPSA is expressed as a function of logP. Compounds located in the red square are likely to cause toxicity. Estimated toxicities of compounds 13 and 19 (blues point) fall within the light green area. (For interpretation of the references to color in this figure legend, the reader is referred to the Web version of this article.) 